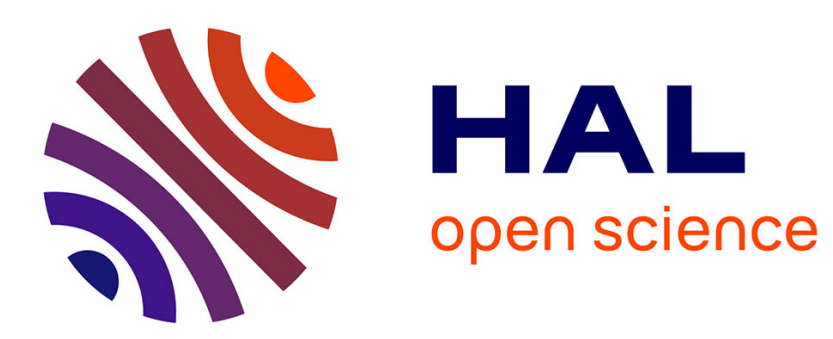

\title{
Flow near porous media boundaries including inertia and slip: A one-domain approach
}

\author{
F. J Valdés-Parada, Didier Lasseux
}

\section{To cite this version:}

F. J Valdés-Parada, Didier Lasseux. Flow near porous media boundaries including inertia and slip: A one-domain approach. Physics of Fluids, 2021, 33 (7), pp.073612. 10.1063/5.0056345 . hal-03289702

\section{HAL Id: hal-03289702 \\ https://hal.science/hal-03289702}

Submitted on 18 Jul 2021

HAL is a multi-disciplinary open access archive for the deposit and dissemination of scientific research documents, whether they are published or not. The documents may come from teaching and research institutions in France or abroad, or from public or private research centers.
L'archive ouverte pluridisciplinaire HAL, est destinée au dépôt et à la diffusion de documents scientifiques de niveau recherche, publiés ou non, émanant des établissements d'enseignement et de recherche français ou étrangers, des laboratoires publics ou privés. 


\title{
Flow near porous media boundaries including inertia and slip: A one-domain approach
}

\author{
Phys. Fluids 33, 073612 (2021) \\ doi: 10.1063/5.0056345
}

\section{F. J. Valdés-Parada ${ }^{\top}$ (D) and D. Lasseux ${ }^{2, a)}$ (D)}

\begin{abstract}
AFFILIATIONS
'Departamento de Ingeniería de Procesos e Hidráulica, Universidad Autónoma Metropolitana-Iztapalapa, Av. San Rafael Atlixco 186, 09340 Ciudad de México, Mexico

${ }^{2}$ I2M, UMR 5295, CNRS, Univ. Bordeaux, 351, Cours de la Libération, 33405 Talence CEDEX, France
\end{abstract}

a) Author to whom correspondence should be addressed: didier.lasseux@u-bordeaux.fr

\begin{abstract}
This work addresses the macroscopic modeling of flow near porous media boundaries. This includes the vicinity with a fluid channel (i.e., a fracture), another rigid porous medium, or an impervious non-deformable solid. The analysis is carried out for one-phase, steady, incompressible, inertial, and isothermal flow of a Newtonian fluid, considering slip effects at the solid-fluid interfaces. A one-domain approach is proposed, employing a simplified version of the volume averaging method, while conceiving the system as two homogeneous regions separated by an inter-region. The upscaling procedure yields a closed macroscopic model including a divergence-free average (filtration) velocity for the mass balance equation and a unique momentum equation having a Darcy structure. The latter involves apparent permeability tensors that are constant in the homogeneous regions and position-dependent in the inter-region. All the permeability tensors are determined from the solution of coupled closure problems that are part of the developments. The derived model is validated by comparisons with direct numerical simulations in several two-dimensional configurations, namely, two porous media of contrasted properties in direct contact or separated by a fracture, the boundaries being either flat or wavy and a porous medium in contact with a flat or corrugated solid wall or separated from the latter by a fluid layer. The simplicity and versatility of the derived model make it an interesting alternative to existing one- and twodomain approaches developed so far.
\end{abstract}

\section{INTRODUCTION}

Modeling flow at the macroscopic scale in the vicinity of surfaces bounding a porous medium is of major interest, both from fundamental and applications points of view, although it remains a challenging difficulty, which has not received a definite solution yet. Since the pioneering work of Beavers and Joseph, and during the past 50 years, attention has been widely focused on the eponym configuration to study fluid motion in the inter-region separating a porous medium and a fluid channel, itself bounded by a solid flat plane, from theoreti$\mathrm{cal}^{2-10}{ }^{2-x p e r i m e n t a l},{ }^{11-14}$ and numerical ${ }^{15-20}$ points of view. As recalled in a recent work, ${ }^{10}$ two methods have been followed for this purpose, both having advantages and drawbacks. In the first one, referred to as the one-domain approach (ODA), a single macroscopic transport equation is sought with abruptly but continuously varying effective coefficients in the inter-region. The second one consists of two separate equations in the free fluid (Navier-Stokes equation) and in the porous region (Darcy or Darcy-Brinkman-type equation) that are applied on each side of a dividing surface. The latter approach requires boundary conditions at the dividing surface, which, in addition, needs to be adequately positioned.

Attention has also been paid to flow description in the vicinity of boundaries for other configurations like, for instance, a porous medium in contact with a solid or with another porous medium of contrasted characteristics. In the former case, a boundary condition at the wall was derived using the homogenization theory on the Stokes equation $^{21}$ or a matched asymptotic expansion method, assuming that the Darcy-Brinkman equation is valid everywhere in the porous zone. $^{22}$ In both analyses, a boundary condition was obtained under the form of a jump in the tangential velocity at the solid wall. Considering the particular case of a bundle of parallel tubes as a model porous medium, the average velocity profile was analyzed in the vicinity of a solid impermeable wall, showing the importance of the averaging domain size in the macroscopic description. ${ }^{23}$ For flow involving two porous media in contact, the two-domain approach is usually adopted, making use of Darcy's law on both sides of a dividing surface and continuity of the average pressure and flux. ${ }^{24} \mathrm{~A}$ similar approach 
is also used at the fracture-matrix boundary in fractured porous media. ${ }^{25,26}$ Many other problems where the boundary with a porous medium plays a central role have been recently investigated, like, for instance, flow near a porous membrane, ${ }^{27}$ lubrication in the presence of a porous material, ${ }^{28,29}$ or coupling with mass transport. ${ }^{30}$ So far, however, a unified methodology to provide a macroscopic flow description in the bulk of a porous medium and close to its boundary, whatever its nature and shape, is still lacking, albeit of valuable interest.

The purpose of the present analysis is hence to propose a general framework that makes use of a one-domain approach to obtain a closed macroscopic model consisting of a single mass and a single momentum equation describing flow in a system involving a porous medium limited by boundaries of different types. This is carried out for one-phase steady, isothermal, inertial, and incompressible flow of a Newtonian fluid. In addition, the possible existence of slip effects at the solid-fluid interfaces is considered in the developments. The method is inspired from that reported by Valdés-Parada and Lasseux, ${ }^{10}$ which was restricted to the Beavers and Joseph configuration. In this sense, the present work extends the upscaling approach to many other very different situations.

As a generic configuration, the heterogeneous system is conceived as two porous media or one porous medium and a solid (all of them being non-deformable materials), which may be in direct contact or separated by a fluid channel. In the latter case, this corresponds to a heterogeneous fractured porous medium or to the Beavers and Joseph configuration if one of the two media is impervious with a channel that may not have flat parallel walls. Indeed, no restriction regarding the shape of the separation zone between the media is assumed, a priori. The analysis is performed by considering three distinct regions as schematically represented in Fig. 1, namely,

- the homogeneous parts of the system, referred to as the $\omega_{1}$ - and $\omega_{2}$-regions in the bulk of the two media;

- the $\omega$-inter-region, which corresponds to the zone where boundary layers develop. When the system embeds a fluid channel between the two media, this free fluid zone may be included within this region.

On this basis, the article is organized as follows. In Sec. II, the pore-scale flow problem is presented. An upscaling procedure is applied on it to derive the closed macroscopic mass and momentum equations and this is detailed in Sec. III. The results of the upscaling procedure are a single macroscopic mass balance equation and a Darcy-like equation for momentum transport. The latter is written in terms of an effective apparent permeability, which is a position-

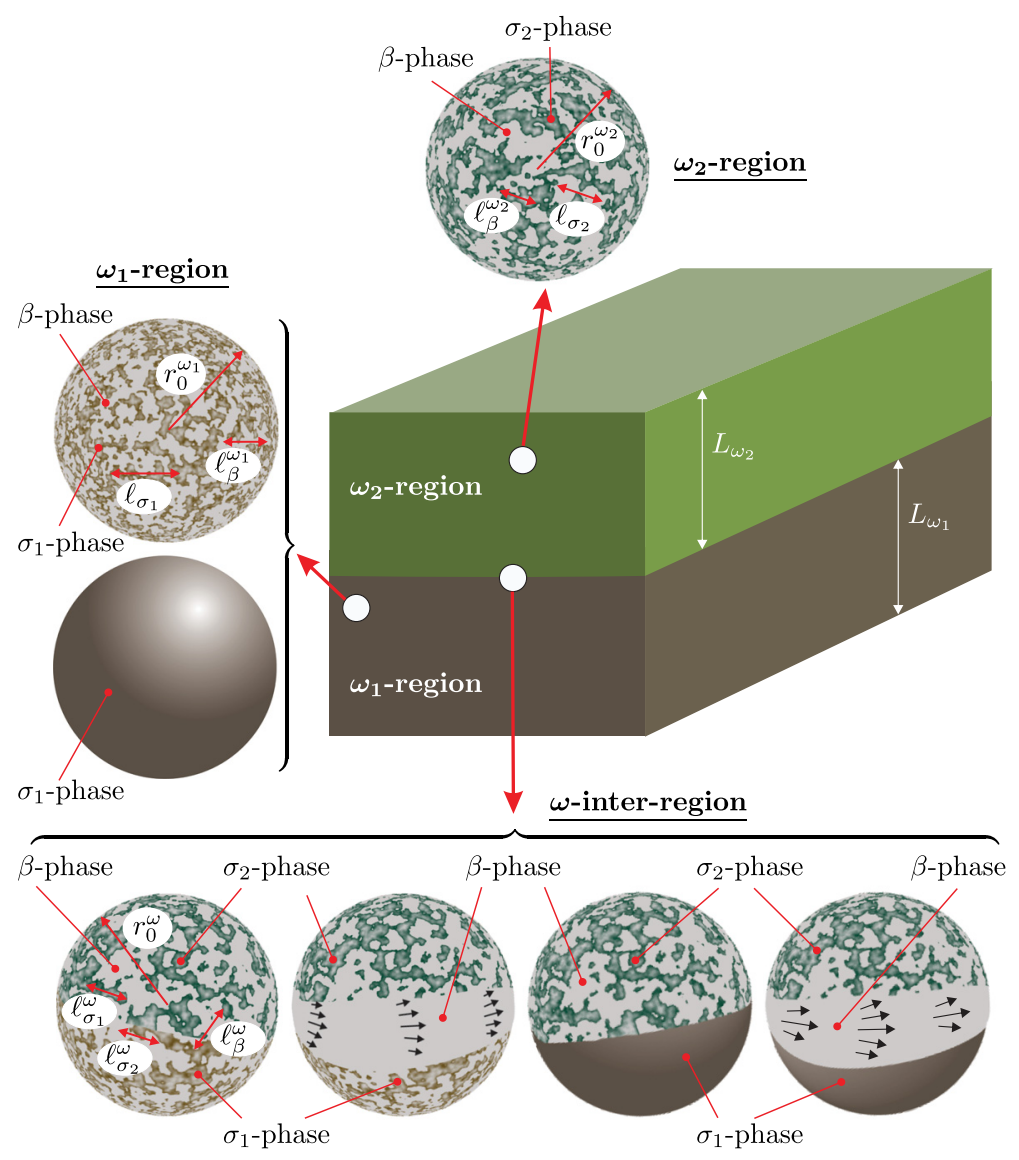

FIG. 1. Schematic representation of the system under consideration including two porous media or a porous medium and an impervious solid in direct contact or separated by a fluid channel (fracture). The two homogeneous regions, $\omega_{1}$ and $\omega_{2}$, of characteristic macroscopic size $L_{\omega_{1}}$ and $L_{\omega_{2}}$, are separated by the $\omega$-inter-region, which may include a fluid channel. The solid phase is $\sigma_{i}$ in each medium $i, i=1,2$, whereas the $\beta$-phase denotes the fluid-phase everywhere. The averaging domain in each region is of characteristic dimension $r_{0}^{\kappa}, \kappa=\omega_{1}, \omega_{2}, \omega$, while the characteristic pore-size is, respectively, $\ell_{1}^{\sigma}, \ell_{2}^{\sigma}$, and $\ell_{\sigma_{1}}^{\omega}$. 
dependent tensor that can be determined from closure problems to be solved in the three regions: $\omega_{1}, \omega_{2}$, and $\omega$. Section IV is dedicated to some illustrative results in four distinct cases, namely, two different porous media sharing a boundary, two porous materials embedding a fracture, and a porous medium in contact with a non-percolating one (or a solid) or separated from it by a fracture. The upscaled model is validated from comparisons with direct numerical simulations (DNS). Conclusions are drawn in Sec. V.

\section{PORE-SCALE PROBLEM}

The system under consideration is sketched in Fig. 1 showing the two homogeneous regions, $\omega_{1}$ and $\omega_{2}$, of the two media (one being potentially non-percolating or, equivalently, an impervious solid) and the $\omega$-inter-region, which may include a fluid channel. The Newtonian fluid saturating the system is the $\beta$-phase while the rigid solid phases constitutive of the two media are denoted by $\sigma_{1}$ and $\sigma_{2}$, respectively. Since flow is assumed to be steady, incompressible, laminar, and isothermal with possible slip effects at the solid-fluid interfaces, the pore-scale governing equations for the flow in the entire system are given by $\left(\kappa\right.$ denotes the $\omega_{1^{-}}, \omega_{2^{-}}$, or $\omega$-region)

$$
\begin{gathered}
\nabla \cdot \mathbf{v}_{\kappa}=0 \quad \text { in the } \beta \text {-phase } \\
\rho \mathbf{v}_{\kappa} \cdot \nabla \mathbf{v}_{\kappa}=-\nabla p_{\kappa}+\mu \nabla^{2} \mathbf{v}_{\kappa} \quad \text { in the } \beta \text {-phase } \\
\mathbf{v}_{\kappa}=-\xi_{i} \lambda(\mathbf{I}-\mathbf{n n}) \cdot\left[\mathbf{n} \cdot\left(\nabla \mathbf{v}_{\kappa}+\nabla \mathbf{v}_{\kappa}^{T}\right)\right] \text { at } \mathscr{A}_{\beta \sigma_{i}}^{\kappa}, i=1,2
\end{gathered}
$$

In the above equations, $\rho$ and $\mu$ represent the fluid density and viscosity that are both assumed constant. In addition, $\mathbf{v}_{\kappa}$ and $p_{\kappa}$ denote the fluid velocity and pressure in the $\kappa$-region. Without any lack of generality, volume forces are not explicitly considered in the momentum transport equation and may be included in the definition of the pressure. The boundary condition in Eq. (1c) at the interface, $\mathscr{A}_{\beta \sigma_{\sigma}}^{\kappa}$, between the fluid and the solid phase, $\sigma_{i}$ in the $\kappa$-region, is written as a first-order Navier-type slip condition in the context of rarefied effects. $^{31-33}$ Here, $\mathbf{I}$ is the identity tensor and $\mathbf{n}$ is the unit normal vector directed from the $\beta$-phase toward the $\sigma_{i}$-phase. In addition, $\lambda$ denotes the mean free path of the fluid molecules and $\xi_{i}$ is a constant coefficient that is specific to the surface of the solid-phase $\sigma_{i}$ and the $\beta$-phase. It is defined in terms of the tangential momentum accommodation coefficient, $\alpha_{i}$, as follows:

$$
\xi_{i}=\frac{\left(2-\alpha_{i}\right)}{\alpha_{i}}, \quad i=1,2 .
$$

Because of the incompressible flow assumption, $\lambda$ is treated as a constant in the developments that follow. It should be noted that the same type of slip boundary condition could be written while considering it as an effective one resulting from a pre-upscaling of a no-slip condition at rough surfaces. ${ }^{34}$

Macroscopic boundary conditions must be provided to close the problem; however, they are left unspecified at this point as they will not be used in the developments that follow.

An upscaling procedure shall now be applied on the above boundary value problem in order to obtain the macroscopic closed model.

\section{UPSCALING}

\section{A. Preliminaries}

Different techniques could be used to derive the upscaled model, as, for instance, double-scale homogenization ${ }^{35}$ or volume averaging. ${ }^{36}$ In this work, the latter is employed in a shortened version. The averaging process is carried out by making use of an averaging domain, $\mathscr{V}_{\kappa}$, of measure $V_{\kappa}$ and characteristic size $r_{0}^{\kappa}$ in the $\kappa$-region $\left(\kappa=\omega_{1}, \omega_{2}, \omega\right)$. For any physical quantity, $\psi_{\kappa}$, in this region, the superficial and intrinsic averaging operators are then defined as ${ }^{36}$

$$
\begin{aligned}
\left\langle\psi_{\kappa}\right\rangle_{\kappa} & =\frac{1}{V_{\kappa}} \int_{\mathscr{\gamma}_{\beta \kappa}} \psi_{\kappa} d V, \\
\left\langle\psi_{\kappa}\right\rangle_{\kappa}^{\beta} & =\frac{1}{V_{\beta \kappa}} \int_{\mathscr{\gamma}_{\beta \kappa}} \psi_{\kappa} d V .
\end{aligned}
$$

Here $\mathscr{V}_{\beta \kappa}$ (of volume $V_{\beta \kappa}$ ) represents the portion of $\mathscr{V}_{\kappa}$ occupied by the fluid phase in the averaging domain located in the $\kappa$-region. The two averages are related by

$$
\left\langle\psi_{\kappa}\right\rangle_{\kappa}=\frac{V_{\beta \kappa}}{V_{\kappa}}\left\langle\psi_{\kappa}\right\rangle_{\kappa}^{\beta}
$$

The ratio $V_{\beta \kappa} / V_{\kappa}$ is position-dependent in the inter-region $(\kappa=\omega)$. For $\kappa=\omega_{i}(i=1,2)$, and provided $\mathscr{V}_{\kappa}$ is chosen to be representative, i.e., so as to contain all the necessary structural information of the material, this ratio is the porosity, $\varepsilon_{\omega_{i}}$, of the corresponding homogeneous region, which is supposed to be constant in the remainder of this work.

\section{B. Average mass balance}

To derive the average form of the mass balance equation valid everywhere in the system, the superficial average operator is applied to the mass balance equation (1a). In order to interchange spatial differentiation and integration, it is convenient to use the averaging theorem (or Leibniz rule), which, for the divergence operator, writes ${ }^{37}$

$$
\left\langle\nabla \cdot \psi_{\kappa}\right\rangle_{\kappa}=\nabla \cdot\left\langle\psi_{\kappa}\right\rangle_{\kappa}+\frac{1}{V_{\kappa}} \int_{\mathscr{A}_{\beta \sigma_{i}}^{\kappa}} \mathbf{n} \cdot \psi_{\kappa} d A .
$$

Once this theorem is employed, the average mass equation in the $\kappa$ region $\left(\kappa=\omega_{1}, \omega_{2}, \omega\right)$ can be written as

$$
\nabla \cdot\left\langle\mathbf{v}_{\kappa}\right\rangle_{\kappa}+\frac{1}{V_{\kappa}} \int \mathbf{n} \cdot \mathbf{v}_{\kappa} d A=0 .
$$

Since the velocity is tangential to this solid-fluid interfaces (no mass is transferred between the solid and fluid phases), the last term is zero in the above equation, which reduces to

$$
\nabla \cdot\left\langle\mathbf{v}_{\kappa}\right\rangle_{\kappa}=0 .
$$

This represents the closed form of the macroscopic mass equation valid in all the three regions of the system and it does not require any further developments. 


\section{Average momentum balance}

\section{Homogeneous regions, $\omega_{1}$ and $\omega_{2}$}

The derivation of the macroscopic momentum equations in $\omega_{i}$ $(i=1,2)$, for flow including inertia and slip effects, can be found in previously reported works. ${ }^{39-42}$ Here, the same steps as those presented in a recent article by Valdés-Parada and Lasseux, ${ }^{10}$ to which the reader is referred to for more details, are followed. For the sake of brevity, it suffices here to only recall a summary of the procedure to reach the result.

First, the $\omega_{i}$-regions are conceived as periodic structures and the averaging domain is taken as a Representative Elementary Volume (REV). ${ }^{43}$ To be representative, this domain must contain all the necessary structural information and is hence constrained by the hierarchy

$$
\ell_{\omega_{i}} \ll r_{0}^{\omega_{i}}
$$

where $\ell_{\omega_{i}}=\max \left(\ell_{\beta}^{\omega_{i}}, \ell_{\sigma_{i}}\right), \ell_{\beta}^{\omega_{i}}$, and $\ell_{\sigma_{i}}$, respectively, denoting the characteristic size of the pores and of the solid phase $i$ in $\omega_{i}$-region (see Fig. 1). In some practical situations, the constraint in (6a) is overly severe and can be relaxed to $\ell_{\omega_{i}}<r_{0}^{\omega_{i}}$. Moreover, it is assumed that, for both regions, the lattice vectors of the periodic unit cells are the same and that a unique size of the REV can be chosen so as to contain at least one of the largest periodic geometrical unit cell of the two regions. Finally, the classical assumption of length-scale separation is also retained, i.e.,

$$
r_{0}^{\omega_{i}} \ll L_{\omega_{i}}
$$

where $L_{\omega_{i}}$ is the minimum dimension of each of the porous media in the three directions of space. form $^{44}$

Next, the pressure gradient in $\omega_{i}$ is decomposed under the

$$
\nabla p_{\omega_{i}}=\nabla\langle p\rangle_{\omega_{i}}^{\beta}+\nabla \tilde{p}_{\omega_{i}}
$$

$\tilde{p}_{\omega_{i}}$ being the pressure deviations in $\omega_{i}$, and, for simplification in the notation, $\nabla\langle p\rangle_{\omega_{i}}^{\beta} \equiv \nabla\left\langle p_{\omega_{i}}\right\rangle_{\omega_{i}}^{\beta}$. As a consequence of the length-scale constraint expressed in (6b), $\nabla\langle p\rangle_{\omega_{i}}^{\beta}$ is treated as a constant in the REV. Moreover, $\mathbf{v}_{\omega_{i}}$ and $\tilde{p}_{\omega_{i}}$ can be regarded as periodic variables at the boundaries of the REV so that the flow problem is reformulated as (here, $i=1,2$ )

$$
\begin{gathered}
\nabla \cdot \mathbf{v}_{\omega_{i}}=0 \quad \text { in the } \beta \text {-phase, } \\
\frac{\rho}{\mu} \mathbf{v}_{\omega_{i}} \cdot \nabla \mathbf{v}_{\omega_{i}}=-\frac{1}{\mu} \nabla \tilde{p}_{\omega_{i}}+\nabla^{2} \mathbf{v}_{\omega_{i}}-\frac{1}{\mu} \nabla\langle p\rangle_{\omega_{i}}^{\beta} \\
\text { in the } \beta \text {-phase, } \\
\mathbf{v}_{\omega_{i}}=-\xi_{i} \lambda(\mathbf{I}-\mathbf{n n}) \cdot\left[\mathbf{n} \cdot\left(\nabla \mathbf{v}_{\omega_{i}}+\nabla \mathbf{v}_{\omega_{i}}^{T}\right)\right] \text { at } \mathscr{A}_{\beta \sigma_{i}}^{\omega_{i}}, \\
\psi\left(\mathbf{r}+\mathbf{l}_{j}\right)=\psi(\mathbf{r}), \quad j=1,2,3 ; \psi=\mathbf{v}_{\omega_{i}}, \tilde{p}_{\omega_{i}}, \\
\left\langle\tilde{p}_{\omega_{i}}\right\rangle_{\omega_{i}}^{\beta}=0 .
\end{gathered}
$$

The last equation results from the fact that $\left\langle p_{\omega_{i}}\right\rangle^{\beta}$ can be treated as a constant within the REV and is required for the problem to be wellposed. In the periodic conditions [Eq. (8d)], $\mathbf{l}_{j}$ represents the common periodic lattice vectors for the two regions. It should be noted that, here, distinct pressure gradients are considered in the different regions in contrast with a previous work where $\nabla\langle p\rangle_{\omega_{i}}^{\beta}$ was taken as a constant all through the system. ${ }^{10}$

Considering the convective velocity as a known field in the inertial term in Eq. ( $8 b)$ so that the problem can be seen as linear, and keeping in mind that $\nabla\langle p\rangle_{\omega_{i}}^{\beta}$ is constant within the REV, the formal solution of this boundary value problem can be written as $^{10}$

$$
\begin{gathered}
\mathbf{v}_{\omega_{i}}=-\frac{\mathbf{D}_{\omega_{i}}}{\mu} \cdot \nabla\langle p\rangle_{\omega_{i}}^{\beta}, \\
\tilde{p}_{\omega_{i}}=-\mathbf{d}_{\omega_{i}} \cdot \nabla\langle p\rangle_{\omega_{i}}^{\beta} .
\end{gathered}
$$

The reader is referred to previously reported works ${ }^{39,42,45}$ for details about the derivation of this solution. Here, $\mathbf{D}_{\omega_{i}}$ and $\mathbf{d}_{\omega_{i}}$ are closure variables that map the macroscopic pressure gradient onto the velocity and pressure deviations in each homogeneous region. These variables solve the following boundary value problems $(i=1,2)$ :

Problem- $\omega_{i}$

$$
\begin{gathered}
\nabla \cdot \mathbf{D}_{\omega_{i}}=\mathbf{0} \quad \text { in the } \beta \text {-phase } \\
-\frac{\rho}{\mu^{2}} \nabla\langle p\rangle_{\omega_{i}}^{\beta} \cdot \mathbf{D}_{\omega_{i}}^{T} \cdot \nabla \mathbf{D}_{\omega_{i}}=-\nabla \mathbf{d}_{\omega_{i}}+\nabla^{2} \mathbf{D}_{\omega_{i}}+\mathbf{I} \\
\text { in the } \beta \text {-phase, } \\
\mathbf{D}_{\omega_{i}}=-\xi_{i} \lambda(\mathbf{I}-\mathbf{n n}) \cdot\left[\mathbf{n} \cdot\left(\nabla \mathbf{D}_{\omega_{i}}+\nabla \mathbf{D}_{\omega_{i}}^{T 1}\right)\right] \quad \text { at } \mathscr{A}_{\beta \sigma_{i}}^{\omega_{i}}, \\
\psi\left(\mathbf{r}+\mathbf{l}_{j}\right)=\psi(\mathbf{r}), \quad j=1,2,3 ; \psi=\mathbf{D}_{\omega_{i}}, \mathbf{d}_{\omega_{i}}, \\
\left\langle\mathbf{d}_{\omega_{i}}\right\rangle_{\omega_{i}}^{\beta}=\mathbf{0} .
\end{gathered}
$$

In the boundary condition at the solid-fluid interfaces [Eq. (10c)], the superscript $T 1$ denotes the transpose of a third-order tensor that permutes the first and second indices, i.e., $(\nabla D)_{i j k}^{T 1}=(\nabla D)_{j i k}$.

The macroscopic momentum equation can then be obtained by applying the superficial averaging operator to the solution for $\mathbf{v}_{\omega_{i}}$ given in Eq. (9a). This yields

$$
\left\langle\mathbf{v}_{\omega_{i}}\right\rangle_{\omega_{i}}=-\frac{\mathbf{H}_{\omega_{i}}}{\mu} \cdot \nabla\langle p\rangle_{\omega_{i}}^{\beta},
$$

with

$$
\mathbf{H}_{\omega_{i}}=\left\langle\mathbf{D}_{\omega_{i}}\right\rangle_{\omega_{i}}
$$

being the apparent permeability tensor that is specific to the $\omega_{i}$-region and whose symmetry properties, in the absence of slip, were reported in a previous work. ${ }^{46}$ When there is no inertial nor slip effects, $\mathbf{H}_{\omega_{i}}$ reduces to the intrinsic permeability tensor. Note that if $\omega_{i}$ is a nonpercolating medium (i.e., a solid), the corresponding closure problem degenerates $\left(\mathbf{D}_{\omega_{i}}=\mathbf{0}, \mathbf{H}_{\omega_{i}}=\mathbf{0}, \mathbf{v}_{\omega_{i}}=\left\langle\mathbf{v}_{\omega_{i}}\right\rangle_{\omega_{i}}=\mathbf{0}\right.$, and $p_{\omega_{i}}=$ const is the trivial solution) and can hence be ignored.

The derivation of the average momentum equation must now be carried out in the $\omega$-inter-region to complete the macroscopic model. 


\section{2. $\omega$-inter-region}

This region is delimited by two fictitious dividing planes denoted as $\mathscr{A}_{\omega_{i}}(i=1,2)$, corresponding to the locations where the assumption of spatial homogeneity breaks down as illustrated in Fig. 2. These planes are defined by two vectors of the periodic lattice, $\mathbf{l}_{1}$ and $\mathbf{l}_{2}$. At these boundaries, it is physically relevant to impose that the pore-scale velocity is continuous. Once the closure problem- $\omega_{i}(i=1,2)$ is solved in the REV, the fields of $\mathbf{D}_{\omega_{i}}$ are available and so is the pore-scale velocity at $\mathscr{A}_{\omega_{i}}$, which is given by its expression in Eq. (9a). As a consequence, the pore-scale problem in the $\omega$-inter-region can be stated as follows:

$$
\begin{gathered}
\nabla \cdot \mathbf{v}_{\omega}=0 \quad \text { in the } \beta \text {-phase } \\
\frac{\rho}{\mu} \mathbf{v}_{\omega} \cdot \nabla \mathbf{v}_{\omega}=-\frac{1}{\mu} \nabla \tilde{p}_{\omega}+\nabla^{2} \mathbf{v}_{\omega}-\frac{1}{\mu} \nabla\langle p\rangle_{\omega}^{\beta} \\
\text { in the } \beta \text {-phase, } \\
\mathbf{v}_{\omega}=-\xi_{i} \lambda(\mathbf{I}-\mathbf{n n}) \cdot\left[\mathbf{n} \cdot\left(\nabla \mathbf{v}_{\omega}+\nabla \mathbf{v}_{\omega}^{T}\right)\right] \\
\text { at } \mathscr{A}_{\beta \sigma_{i}}^{\omega}, \quad i=1,2, \\
\mathbf{v}_{\omega}=-\frac{\mathbf{D}_{\omega_{i}}}{\mu} \cdot \nabla\langle p\rangle_{\omega_{i}}^{\beta} \text { at } \mathscr{A}_{\omega_{i}}, i=1,2, \\
\psi\left(\mathbf{r}+\mathbf{l}_{j}\right)=\psi(\mathbf{r}), \quad j=1,2 ; \psi=\mathbf{v}_{\omega}, \tilde{p}_{\omega}, \\
\left\langle\tilde{p}_{\omega}\right\rangle_{\omega}^{\beta}=0 .
\end{gathered}
$$

The pressure decomposition introduced in the momentum equation (13b) makes use of an averaging domain which size, $r_{0}^{\omega}$, is supposed to satisfy

$$
\ell_{\omega} \ll r_{0}^{\omega}
$$

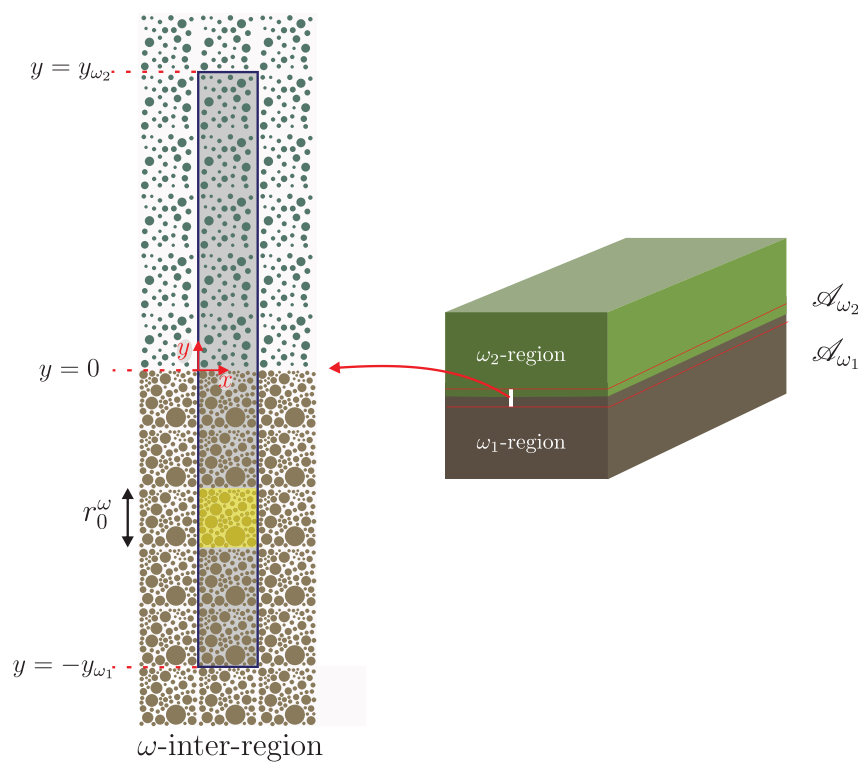

FIG. 2. Sketch of the inter-region separating two porous media. This region is comprised between $y=-y_{\omega_{1}}$ (locating $\mathscr{A}_{\omega_{1}}$ ) and $y=y_{\omega_{2}}$ (locating $\mathscr{A}_{\omega_{2}}$ ). The yellow square represents the averaging domain of size $r_{0}^{(\omega)}$ and its centroid can be located anywhere in the inter-region. where $\ell_{\omega}=\max \left(\ell_{\beta}^{\omega}, \ell_{\sigma_{i}}^{\omega}\right), \ell_{\beta}^{\omega}$, and $\ell_{\sigma_{i}}^{\omega}$, respectively, denoting the characteristic size of the pores and of the solid phase $i$ in the $\omega$-inter-region (see Fig. 1). It should be noted that the section size of the averaging domain in the plane $\mathscr{A}_{\omega_{i}}(i=1,2)$ must be compliant with that of the REV in the corresponding adjacent homogeneous region. The procedure to determine the location of $\mathscr{A}_{\omega_{i}}$ will be clearly stated later on.

A simple formal solution to the above pore-scale problem can be proposed upon assuming, first, that the convective velocity in the inertial term of the momentum equation (13b) is a known field. With this hypothesis, the problem can be considered as linear. Second, since this problem is made non homogeneous by the presence of three macroscopic sources, namely, $\nabla\langle p\rangle_{\omega_{i}}^{\beta},(i=1,2)$, and $\nabla\langle p\rangle_{\omega}^{\beta}$, the formal solution can be sought as a linear combination of the three sources, provided they can be treated as constants in the $\omega$-inter-region. By definition, the two sources at $\mathscr{A}_{\omega_{i}}$ take values equal to those in the REV adjacent to this boundary in the $\omega_{i}$-region and have hence given constant values. However, treating $\nabla\langle p\rangle_{\omega}^{\beta}$ as a constant is subject to a constraint that can be expressed as

$$
\delta_{\omega} \ll L_{p},
$$

where $\delta_{\omega}$ and $L_{p}$, respectively, represent the maximum size of the $\omega$ inter-region in the three directions of space and the minimum characteristic length over which $\nabla\langle p\rangle_{\omega}^{\beta}$ experiences significant variations. Under these circumstances, the solution for $\mathbf{v}_{\omega}$ and $\tilde{p}_{\omega}$ can be formally written as

$$
\begin{gathered}
\mathbf{v}_{\omega}=-\frac{\mathbf{F}_{\omega_{i}}}{\mu} \cdot \nabla\langle p\rangle_{\omega_{i}}^{\beta}-\frac{\mathbf{F}_{\omega}}{\mu} \cdot \nabla\langle p\rangle_{\omega}^{\beta}, \\
\tilde{p}_{\omega}=-\mathbf{f}_{\omega_{i}} \cdot \nabla\langle p\rangle_{\omega_{i}}^{\beta}-\mathbf{f}_{\omega} \cdot \nabla\langle p\rangle_{\omega}^{\beta} .
\end{gathered}
$$

When the aforementioned constraint is not satisfied, the solution for $\mathbf{v}_{\omega}$ and $\tilde{p}_{\omega}$ can be shown to be nonlocal in space and requires the computation of the associated Green's functions problem. This is however beyond the scope of this article. In the above expressions, where the Einstein notation was implicitly used in the first terms on the righthand sides, the closure variables $\mathbf{F}_{\omega_{i}}, \mathbf{f}_{\omega_{i}}(i=1,2)$ and $\mathbf{F}_{\omega}, \mathbf{f}_{\omega}$ are solutions of the following three closure problems:

Problems-I-II $(i=1,2)$,

$$
\begin{gathered}
\nabla \cdot \mathbf{F}_{\omega_{i}}=\mathbf{0} \quad \text { in the } \beta \text {-phase, } \\
\frac{\rho}{\mu} \mathbf{v}_{\omega} \cdot \nabla \mathbf{F}_{\omega_{i}}=-\frac{1}{\mu} \nabla \mathbf{f}_{\omega_{i}}+\nabla^{2} \mathbf{F}_{\omega_{i}} \quad \text { in the } \beta \text {-phase, } \\
\mathbf{F}_{\omega_{i}}=-\xi_{i} \lambda(\mathbf{I}-\mathbf{n n}) \cdot\left[\mathbf{n} \cdot\left(\nabla \mathbf{F}_{\omega_{i}}+\nabla \mathbf{F}_{\omega_{i}}^{T 1}\right)\right] \text { at } \mathscr{A}_{\beta \sigma_{i}}^{\omega}, \\
\mathbf{F}_{\omega_{i}}=\mathbf{D}_{\omega_{i}} \text { at } \mathscr{A}_{\omega_{i}}, \\
\mathbf{F}_{\omega_{i}}=\mathbf{0} \text { at } \mathscr{A}_{\omega_{k}}, \quad k=1,2, k \neq i, \\
\psi\left(\mathbf{r}+\mathbf{l}_{j}\right)=\psi(\mathbf{r}), \quad j=1,2 ; \psi=\mathbf{F}_{\omega_{i}}, \mathbf{f}_{\omega_{i}}, \\
\left\langle\mathbf{f}_{\omega_{i}}\right\rangle_{\omega}^{\beta}=\mathbf{0} .
\end{gathered}
$$

Problem-III,

$$
\begin{gathered}
\nabla \cdot \mathbf{F}_{\omega}=\mathbf{0} \quad \text { in the } \beta \text {-phase, } \\
\frac{\rho}{\mu} \mathbf{v}_{\omega} \cdot \nabla \mathbf{F}_{\omega}=-\nabla \mathbf{f}_{\omega}+\nabla^{2} \mathbf{F}_{\omega}+\mathbf{I} \quad \text { in the } \beta \text {-phase, }
\end{gathered}
$$




$$
\begin{gathered}
\mathbf{F}_{\omega}=-\xi_{i} \lambda(\mathbf{I}-\mathbf{n n}) \cdot\left[\mathbf{n} \cdot\left(\nabla \mathbf{F}_{\omega}+\nabla \mathbf{F}_{\omega}^{T 1}\right)\right] \\
\text { at } \mathscr{A}_{\beta \sigma_{i}}^{\omega}, \quad i=1,2, \\
\mathbf{F}_{\omega}=\mathbf{0} \quad \text { at } \mathscr{A}_{\omega_{i}}, \quad i=1,2, \\
\psi\left(\mathbf{r}+\mathbf{l}_{j}\right)=\psi(\mathbf{r}), \quad j=1,2 ; \quad \psi=\mathbf{F}_{\omega}, \mathbf{f}_{\omega}, \\
\left\langle\mathbf{f}_{\omega}\right\rangle_{\omega}^{\beta}=\mathbf{0} .
\end{gathered}
$$

In addition, the macroscopic momentum equation in the $\omega$ inter-region is obtained by applying the superficial averaging operator to Eq. (16a) in this region, yielding

$$
\left\langle\mathbf{v}_{\omega}\right\rangle_{\omega}=-\frac{\mathbf{H}_{\omega_{i}}^{\omega}}{\mu} \cdot \nabla\langle p\rangle_{\omega_{i}}^{\beta}-\frac{\mathbf{H}_{\omega}^{\omega}}{\mu} \cdot \nabla\langle p\rangle_{\omega}^{\beta},
$$

where, again, the Einstein notation is applied. The effective coefficients, $\mathbf{H}_{\omega_{1}}^{\omega}(i=1,2)$ and $\mathbf{H}_{\omega}^{\omega}$, are apparent permeability tensors given by

$$
\begin{gathered}
\mathbf{H}_{\omega_{i}}^{\omega}=\left\langle\mathbf{F}_{\omega_{i}}\right\rangle_{\omega}, i=1,2, \\
\mathbf{H}_{\omega}^{\omega}=\left\langle\mathbf{F}_{\omega}\right\rangle_{\omega}
\end{gathered}
$$

that are obtained from the solutions of the above closure problems I-II-III. These three closure problems are coupled, as can be readily understood when the expression of $\mathbf{v}_{\omega}$ given in Eq. (16a) is replaced back into Eqs. (17b) and (18b). Moreover, this substitution shows that these problems are closed, i.e., their solutions do not require the predetermination of $\mathbf{v}_{\omega}$ by solving the flow problem.

Closure problems I-II-III are to be solved in the domain having a section size in the fictitious planes, $\mathscr{A}_{\omega_{i}}$, equal to that of the REV in the $\omega_{i}$-region and a height equal to the inter-region thickness, i.e., to the distance between the two planes, given by $y_{\omega_{2}}+y_{\omega_{1}}$ as illustrated in Fig. 2. It must be noted that, in the definitions of the apparent permeability tensors given in Eqs. (20), the superficial average is performed over an averaging domain whose extent across the interregion is smaller than this region size. Therefore, its centroid position is moved between $\mathscr{A}_{\omega_{1}}$ and $\mathscr{A}_{\omega_{2}}$ in order to capture the spatial variations of the three apparent permeability tensors.

In situations for which the macroscopic pressure gradient can be considered as uniform (equal to $\nabla\langle p\rangle^{\beta}$ ) in the entire system, the above closure procedure is considerably simplified. Indeed, the pore-scale problem involves a unique source and leads to a single closure problem given by

Problem- $\omega$

$$
\begin{gathered}
\nabla \cdot \mathbf{F}=\mathbf{0} \quad \text { in the } \beta \text {-phase, } \\
-\frac{\rho}{\mu^{2}} \nabla\langle p\rangle^{\beta} \cdot \mathbf{F}^{T} \cdot \nabla \mathbf{F}=-\nabla \mathbf{f}+\nabla^{2} \mathbf{F}+\mathbf{I} \quad \text { in the } \beta \text {-phase, } \\
\mathbf{F}=-\xi_{i} \lambda(\mathbf{I}-\mathbf{n n}) \cdot\left[\mathbf{n} \cdot\left(\nabla \mathbf{F}+\nabla \mathbf{F}^{T 1}\right)\right] \\
\text { at } \mathscr{A}_{\beta \sigma_{i}}^{\omega}, \quad i=1,2, \\
\mathbf{F}=\mathbf{D}_{\omega_{i}} \text { at } \mathscr{A}_{\omega_{i}}, \quad i=1,2, \\
\psi\left(\mathbf{r}+\mathbf{l}_{j}\right)=\psi(\mathbf{r}), \quad j=1,2 ; \psi=\mathbf{F}, \mathbf{f}, \\
\langle\mathbf{f}\rangle_{\omega}^{\beta}=\mathbf{0} .
\end{gathered}
$$

The above problem can be seen as the sum of the closure problems given in Eqs. (17) and (18). The macroscopic momentum equation in the inter-region reduces to

$$
\left\langle\mathbf{v}_{\omega}\right\rangle_{\omega}=-\frac{\mathbf{H}_{\omega}}{\mu} \cdot \nabla\langle p\rangle^{\beta},
$$

with

$$
\mathbf{H}_{\omega}=\langle\mathbf{F}\rangle_{\omega} .
$$

Note that this last result is also obtained from the sum of Eqs. (20).

As a summary, the upscaled model is composed of the macroscopic mass conservation equation (5) and the macroscopic momentum balance equations

$$
\begin{gathered}
\left\langle\mathbf{v}_{\omega_{i}}\right\rangle_{\omega_{i}}=-\frac{\mathbf{H}_{\omega_{i}}}{\mu} \cdot \nabla\langle p\rangle_{\omega_{i}}^{\beta} \text { in the } \omega_{i} \text {-region, } \quad i=1,2, \\
\left\langle\mathbf{v}_{\omega}\right\rangle_{\omega}=-\frac{\mathbf{H}_{\omega_{i}}^{\omega}}{\mu} \cdot \nabla\langle p\rangle_{\omega_{i}}^{\beta}-\frac{\mathbf{H}_{\omega}^{\omega}}{\mu} \cdot \nabla\langle p\rangle_{\omega}^{\beta} \\
\text { in the } \omega \text {-inter-region, }
\end{gathered}
$$

the Einstein notation being implied in the latter. This allows proposing the following unique macroscopic momentum conservation equation for the one-domain approach developed here that takes the following form:

$$
\langle\mathbf{v}\rangle=-\frac{\mathbf{H}_{i}}{\mu} \cdot \nabla\langle p\rangle_{\omega_{i}}^{\beta}-\frac{\mathbf{H}_{12}}{\mu} \cdot \nabla\langle p\rangle_{\omega}^{\beta}, \quad i=1,2,
$$

with again the Einstein notation implied in the first term on the righthand side. In this equation, the three position-dependent apparent permeability tensors are given by

$$
\mathbf{H}_{i}=\left\{\begin{array}{ll}
\delta_{i j} \mathbf{H}_{\omega_{j}} & \text { in the } \omega_{j} \text {-region, } \\
\mathbf{H}_{\omega_{i}}^{\omega)} & \text { in the } \omega \text {-inter-region, }
\end{array} \quad i, j=1,2 .\right.
$$

$\delta_{i j}$ being the Kronecker delta. In addition,

$$
\mathbf{H}_{12}= \begin{cases}\mathbf{0} & \text { in the } \omega_{i} \text {-region, } \quad i=1,2, \\ \mathbf{H}_{\omega}^{\omega} & \text { in the } \omega \text {-inter-region. }\end{cases}
$$

In the above relationships, $\mathbf{H}_{\omega_{i}}, \mathbf{H}_{\omega_{i}}^{\omega}$, and $\mathbf{H}_{\omega}^{\omega}$ are, respectively, given by Eqs. (12), (20a), and (20b). When the macroscopic pressure gradient can be thought of as being uniform in the entire system, the macroscopic momentum equation in the present one-domain approach has the following simple form: ${ }^{10}$

$$
\langle\mathbf{v}\rangle=-\frac{\mathbf{H}}{\mu} \cdot \nabla\langle p\rangle^{\beta} .
$$

Here, the apparent permeability tensor, $\mathbf{H}$, takes the following values in the three regions:

$$
\mathbf{H}= \begin{cases}\mathbf{H}_{\omega_{i}} & \text { in the } \omega_{i} \text {-region, } \quad i=1,2, \\ \mathbf{H}_{\omega} & \text { in the } \omega \text {-inter-region, }\end{cases}
$$

where $\mathbf{H}_{\omega}$ is defined in Eq. (23).

When the macroscopic pressure gradient is not uniform, the values of $\nabla\langle p\rangle_{\omega_{i}}^{\beta}(i=1,2)$ and $\nabla\langle p\rangle_{\omega}^{\beta}$ shall be determined by solving the following macroscale problem (here, $i=1,2$ ):

$$
\mathbf{H}_{\omega_{i}}^{T}: \nabla \nabla\langle p\rangle_{\omega_{i}}^{\beta}=0 \text { in the } \omega_{i} \text {-region, }
$$




$$
\begin{aligned}
& \mathbf{n} \cdot\left(\mathbf{H}_{\omega_{i}} \cdot \nabla\langle p\rangle_{\omega_{i}}^{\beta}\right)=\mathbf{n} \cdot\left(\mathbf{H}_{\omega_{1}}^{\omega} \cdot \nabla\langle p\rangle_{\omega_{1}}^{\beta}+\mathbf{H}_{\omega_{2}}^{\omega} \cdot \nabla\langle p\rangle_{\omega_{2}}^{\beta}\right. \\
& \left.+\mathbf{H}_{\omega}^{\omega} \cdot \nabla\langle p\rangle_{\omega}^{\beta}\right) \text { at } \mathscr{A}_{\omega_{i}}, \\
& \nabla \cdot\left(\mathbf{H}_{\omega_{1}}^{\omega} \cdot \nabla\langle p\rangle_{\omega_{1}}^{\beta}+\mathbf{H}_{\omega_{2}}^{\omega} \cdot \nabla\langle p\rangle_{\omega_{2}}^{\beta}+\mathbf{H}_{\omega}^{\omega} \cdot \nabla\langle p\rangle_{\omega}^{\beta}\right) \\
& =0 \text { in the } \omega \text {-inter-region, } \\
& \mathscr{G}\left(\langle p\rangle_{\kappa}^{\beta}\right)=0, \quad \kappa=\omega_{i}, \quad \omega \text { at } \partial \mathscr{V}_{M} .
\end{aligned}
$$

The boundary condition given in Eq. (29b) expresses the macroscopic flux continuity at the fictitious boundaries $\mathscr{A}_{\omega_{i}}, \mathbf{n}$ denoting the unit normal vector at these boundaries. Moreover, Eq. (29d) is a formal representation of the macroscopic boundary condition at the bounding surfaces of the entire system denoted by $\partial \mathscr{V}_{M}$. These equations are to be solved in a coupled manner with the closure problems providing the five apparent permeability tensors. It should be noticed that the solution of the above macroscopic problem can be the constant value, $\nabla\langle p\rangle^{\beta}$, in the three regions if the configuration and the functional $\mathscr{G}$ are compliant with this result. Under such circumstances, the solution is decoupled from those of the closure problems.

As a final note, it is of interest to propose a procedure to locate the fictitious boundaries $\mathscr{A}_{\omega_{i}}(i=1,2)$. Their position can be determined in an iterative manner, starting from an initial guess corresponding to the edge of a unit cell in the corresponding region. At each iteration, the average velocity, computed on the REV adjacent to $\mathscr{A}_{\omega_{i}}$ inside the homogeneous $\omega_{i}$-region, is compared to that obtained in the averaging domain positioned next to this boundary inside the $\omega$-inter-region. If the average velocities on both sides of $\mathscr{A}_{\omega_{i}}$ differ from each other by more than a criterion value that is fixed a priori, the corresponding boundary is moved further away inside the homogeneous region and this is repeated until the criterion is satisfied. If the initial guess is such that the criterion is satisfied, the boundary is moved toward the inter-region (i.e., outside the homogeneous region) until the criterion is still fulfilled. In both cases, the boundary is moved by a value corresponding to the size of the REV in the corresponding direction at each iteration.

At this point, it is necessary to evaluate the relevance of the macroscopic model. This is the purpose of the four case studies proposed in Sec. IV.

\section{RESULTS}

The model derived in Sec. III is now tested and validated through DNS in four different situations, namely: two porous media of contrasted properties in direct contact (case 1) or separated by a fracture (case 2), as well as a porous medium in contact with a solid wall (case 3) or separated from it by a fluid channel (case 4). The latter case can be viewed as the Beavers and Joseph configuration, with a notable difference that the solid wall is not necessarily flat, nor the porous medium surface which may hold a roughness having a non-negligible amplitude compared to $\ell_{\omega_{i}}$. For the sake of simplicity, all the simulations were performed in a two-dimensional domain. Moreover, the system is supposed to be of infinite extension in the $x$-direction. As a consequence, the DNS can be carried out in a domain including a single vertical stripe of unit cells as the one sketched in Fig. 2 together with periodic boundary conditions on $\mathbf{v}$ and $\tilde{p}$ in the $x$-direction. The closure problems are solved in the same domain.

The numerical solutions of the pore-scale flow equations and closure problems were carried out using the finite element software
COMSOL Multiphysics 5.6. The direct PARDISO solver included in the software was employed to compute the solutions. In addition, standard convergence analyses in terms of mesh and relative tolerance were performed so that the results presented here are independent of these numerical degrees of freedom. Since the mean flow is along $\mathbf{e}_{x}$, and because the macroscopic pressure gradient is along this direction, the comparison is only made on the $x$-component of $\langle\mathbf{v}\rangle$, requiring to only compute the $x x$ components of the apparent permeability tensors. Furthermore, in order to reduce the number of parameters to be specified in the model evaluation, it is convenient to reformulate the microscale flow problem and the closure problems in terms of the following dimensionless quantities:

$$
\begin{gathered}
\mathbf{r}^{*}=\frac{\mathbf{r}}{\ell_{c}}, \quad \mathbf{v}^{*}=\frac{\mathbf{v}}{v_{r e f}}, \quad p^{*}=\frac{\ell_{c} p}{\mu v_{r e f}}, \quad R e=\frac{\rho v_{r e f} \ell_{c}}{\mu}, \\
\lambda^{*}=\frac{\lambda}{\ell_{c}}, \quad \mathbf{D}_{\omega_{i}}^{*}=\frac{\mathbf{D}_{\omega_{i}}}{\ell_{c}^{2}}, \quad \mathbf{d}_{\omega_{i}}^{*}=\frac{\mathbf{d}_{\omega_{i}}}{\ell_{c}} \quad(i=1,2), \\
\mathbf{F}^{*}=\frac{\mathbf{F}}{\ell_{c}^{2}}, \quad \mathbf{f}^{*}=\frac{\mathbf{f}}{\ell_{c}} .
\end{gathered}
$$

Here, $\ell_{c}$ is the height of the geometrical unit cell and $v_{\text {ref }}=\ell_{c}^{2}\left\|\nabla\langle p\rangle^{\beta}\right\| / \mu$. Under this dimensionless formulation, the only parameters that need to be specified are the cell Reynolds $(R e)$ and Knudsen $\left[\xi_{i} \lambda^{*}(i=1,2)\right]$ numbers for a given geometrical configuration. Indeed, the dimensionless macroscopic pressure gradient has a magnitude equal to 1 . For all the results presented below, $R e=10^{3}, \xi_{1} \lambda^{*}=\xi_{2} \lambda^{*}=\xi \lambda^{*}=0.01$, and $\nabla\left\langle p^{*}\right\rangle^{\beta}=-\mathbf{e}_{x}$. This value of the Reynolds number is convenient as it lies below the first Hopf bifurcation. ${ }^{47}$ In addition, the value of cell Knudsen numbers is within the range of validity of the Navier-Stokes equations. It is worth recalling that the interfacial boundary condition can also be regarded as an effective one over rough surfaces. Taking this into account, it is thus possible to use the same cell Knudsen number in the different media considered in this section.

For the sake of easiness, a unique value of $y_{\omega_{i}}, i=1,2$, common to all the different cases reported below, was chosen, following a simplified version of the iterative algorithm presented above with a convergence criterion equal to $0.1 \%$. In fact, it was verified that $y_{\omega_{i}}=10 \ell_{c}, i=1,2$ was a large enough value that satisfies this criterion in all the cases reported below and was hence employed in all the numerical simulations.

The simulations involving two porous media were performed considering four types of porous structures made of $2 \mathrm{D}$ patterns of parallel cylinders of circular cross section with flow orthogonal to the cylinders axes. For each type, the porosity of the $\omega_{1}$ - and $\omega_{2}$-regions (see Figs. 1 and 2) is, respectively, $\varepsilon_{\omega_{1}}=0.4$ and $\varepsilon_{\omega_{2}}=0.8$. For type I, the cylinders are of uniform size and arranged in a square pattern, whereas for type II, they are placed on a staggered grid in which every two columns of cylinders are shifted by $0.5 \ell_{c}$. For type III, both the cylinder radii and positions inside the unit cell are randomly chosen according to a uniform distribution, achieving the same porosity values as in the previous geometries. The type IV configuration resembles type I, except that the porosity is linearly varied in the vertical direction from $\varepsilon_{\omega_{1}}=0.4$ to $\varepsilon_{\omega_{2}}=0.8$ over 10 unit cells between the $\omega_{1}$ - and $\omega_{2}$-regions. For all the results reported below, the averaging domain size in the vertical direction in the $\omega$-inter-region was taken equal to that of the REV in both homogeneous regions, i.e., $r_{0}^{(\omega *}=1$. 


\section{A. Case 1: Two porous media}

In this case, the four types of porous media were considered. Results of local streamlines patterns obtained from DNS for each of them, and colored by the magnitude of the pore-scale velocity, are reported in Fig. 3. For the first four types of geometries, they are represented within a domain corresponding to 4 unit cells centered in the inter-region, whereas for the structure of type IV, the results are reported in the 10 unit cells in which the porosity is varied. As can be seen in this figure, the frontier between the two porous media is flat for structures of types I, III, and IV, whereas for type II, the dividing surface is wavy as the two structures slightly intermingle. This was achieved by reconstructing a periodic pattern in the horizontal direction obtained by alternatively interchanging a cylinder from one structure to the other at the surface of each media before positioning them on the top of each other.

As expected, the velocity range is larger in the coarser porous structure (the top one) since local flow paths are wider. Inertia can clearly be noticed in structures of types I and IV where recirculation zones are formed behind cylinders in the more permeable areas. For

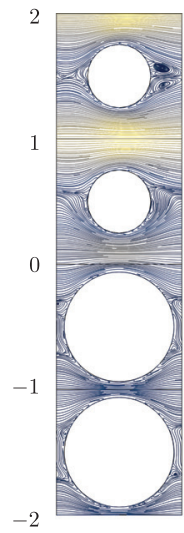

a)

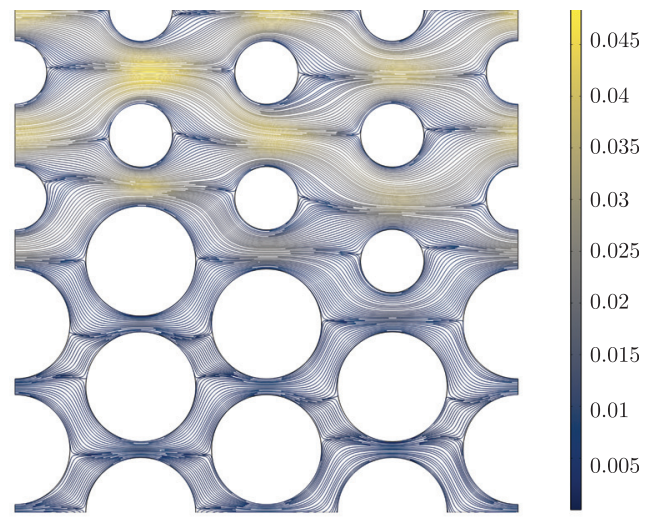

b)

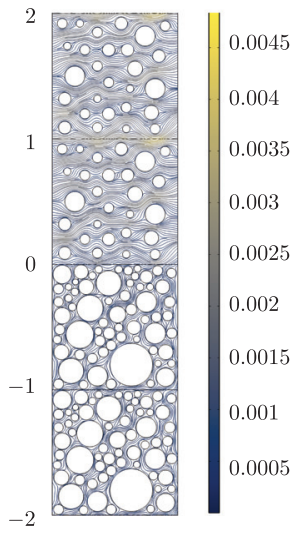

c)

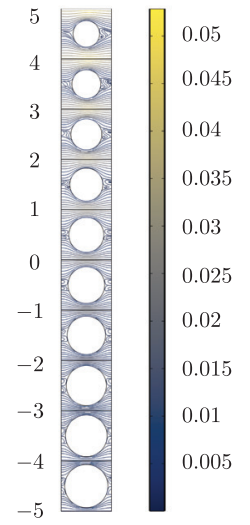

d)
FIG. 3. Examples of the streamlines pattern colored by the dimensionless velocity magnitude near the frontier between two porous media. The different structures shown here correspond to (a) type I, (b) type II, (c) type III, and (d) type IV. In all the simulations, $R e=10^{3}$ and $\xi \lambda^{*}=0.01$ and flow is from left to right. types II and III, recirculations are not observed for this value of Re due to the complicated flow-paths.

The average velocity profiles along the vertical direction, computed from DNS results and obtained from the macroscopic model (ODA), are represented in Fig. 4 for the four types of porous media. In these graphs, the normalized average velocity $\left(\left\langle v_{x}^{*}\right\rangle-\left\langle v_{x}^{*}\right\rangle_{\min }\right) /\left(\left\langle v_{x}^{*}\right\rangle_{\max }-\left\langle v_{x}^{*}\right\rangle_{\min }\right)$, varying between 0 in the $\omega_{1^{-}}$ region and 1 in the coarser $\omega_{2}$-region, was used for convenience in the representation. From Fig. 4(a), it can be noticed that the size of the $\omega$ inter-region could have been limited to $y_{\omega_{1}}^{*}=y_{\omega_{2}}^{*}=2$ for types I, II, and III. For type I, the average velocity experiences a step change centered at $y^{*}=0$ where the porous media frontier is located and smoothly increases with $y^{*}$ on each side of this step. Conversely, for type II, the variation is much smoother but the inter-region tends to thicken due to the more complex structure and the non-smooth frontier between the two porous media. For the type III random structure,

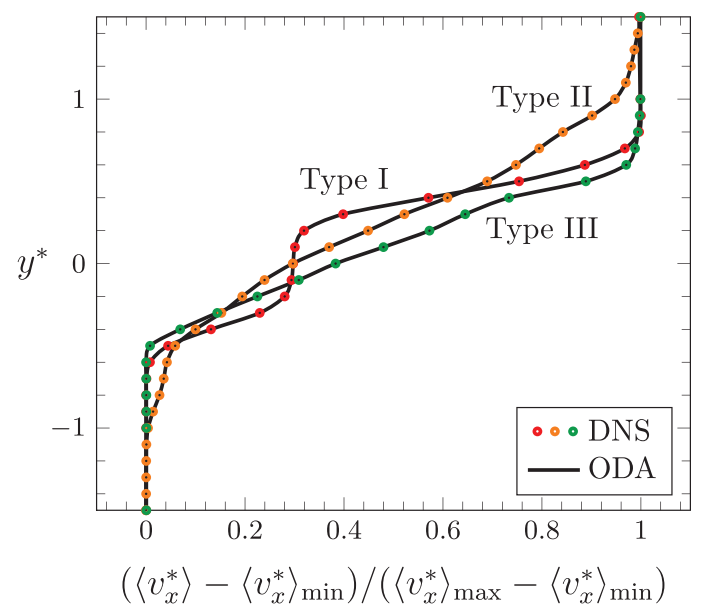

a)

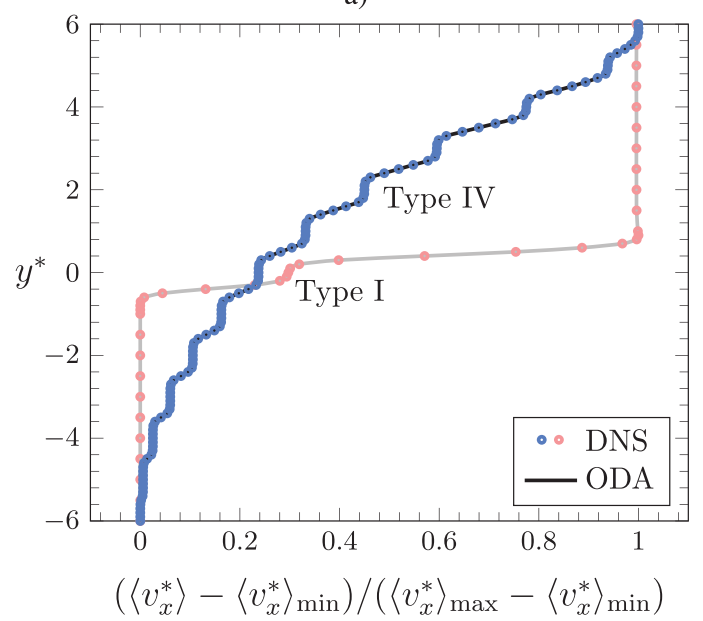

b)

FIG. 4. Average velocity profiles vs the vertical coordinate in the vicinity of the $\omega$ inter-region in the case of two porous media in direct contact. (a) Porous media of types I, II, and II. (b) Porous media of type IV. For comparison purposes, the result obtained with media of type I is recalled in this last figure. 
the average velocity is even smoother but varies over a narrower region, as a result of a flat dividing surface between the two media. Results for type IV, reported in Fig. 4(b), exhibit a succession of step changes of the average velocity while moving the averaging domain from one unit cell to another. Certainly, this results from the choice of the averaging domain size as a smoother profile would be obtained with a larger measure of $r_{0}^{\omega}$. In comparison to type I, whose results are also reported in this figure, the inter-region is much larger as it spans over about 14 unit cells around $y^{*}=0$. This is a direct consequence of the continuously evolving structure over the 10 unit cells between the two homogeneous regions.

For all the configurations, an excellent agreement is achieved between the DNS results and the prediction from the average model derived with the ODA. This shows that, in all cases under consideration here, the upscaled model perfectly captures the average flow characteristics in the inter-region in the case of two porous media in direct contact.

\section{B. Case 2: Two porous media embedding a fracture}

As a second case of analysis, consider now flow between two porous media separated by a fracture. The porous media geometries used in the present case study correspond to the four types studied in case 1 . For types I, III, and IV, the fractures were obtained by removing the solid phase in a geometrical unit cell whose centroid is located at $y=0$ (variant a), $y=-0.5 \ell_{c}$ (variant b), $y=+0.5 \ell_{c}$ (variant c), and, in the final variant $d$, a unit cell of height $2 \ell_{c}$ with its centroid located at $y=0$ was used. For the type II structure, the first and last variants were formed in the same fashion as in the other geometries; however, the second and third variants consisted in removing the last (variant b) and first (variant c) rows of cylinders of the same size, thus giving rise to wavy fractures.

In Figs. 5-7, the dimensionless average velocity profiles, obtained from DNS and predicted from the average model, are reported for structures of types I-III and their variants. These figures also include samples of the pore-scale velocity streamlines colored by the velocity magnitude resulting from DNS. The results corresponding to type IV are not reported since they exhibit a similar behavior to those for the type I structure. Regarding these results, the following comments are in order:

- The presence of fractures translates into a noticeable increment of the fluid velocity, which exceeds the values observed in the previous case. As expected, the average velocity exhibits a parabolic-like profile in the fracture, which connects to the minimal value located at $y \simeq-2 \ell_{c}$ in the homogeneous $\omega_{1}$-region and to the other constant value in the homogeneous $\omega_{2}$-region (at about $y=2 \ell_{c}$ ). The maximum velocity is found near the mid-plane of the fractures. The largest velocity is found in the fourth variant structure, which has the largest fracture aperture. Comparing this value for the different types of structures, it results that the type Id geometry is the one yielding the largest flow-rate, followed by structures of type IIId and IId.

- The average velocity profiles corresponding to the second and third variants of the structures exhibit practically the same amplitudes, with the maximum value shifted according to the location of the fracture mid-plane. The above observations are noted in all the geometries.

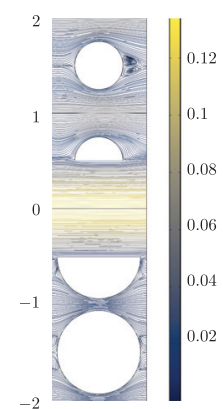

a)

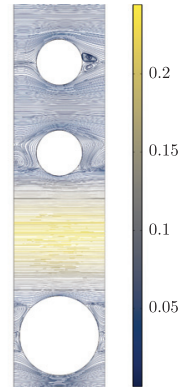

b)

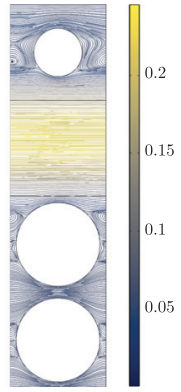

c)

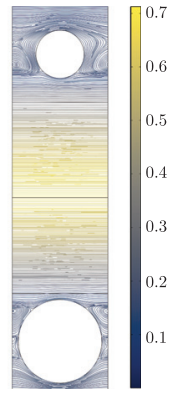

d)

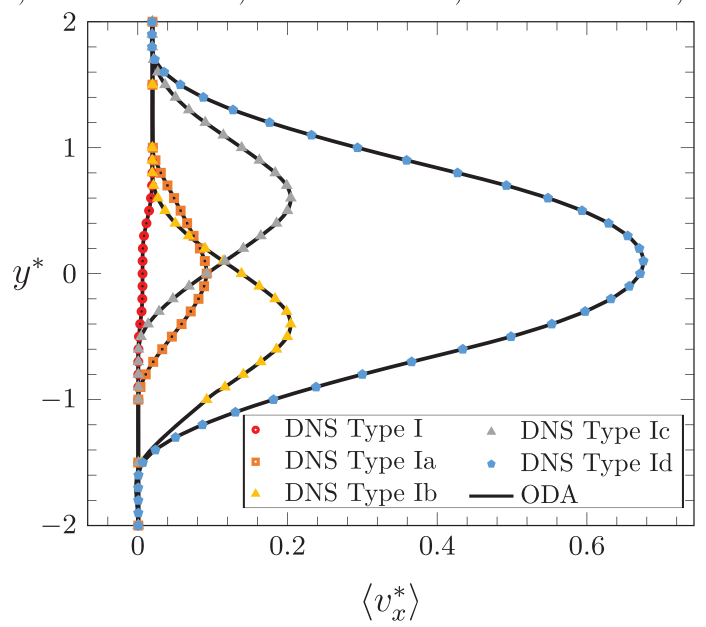

FIG. 5. Top: examples of the streamlines pattern colored by the dimensionless velocity magnitude near the frontier of two porous media separated by a fracture. The structures correspond to the four variants considered for the type I: (a) type la, (b) type lb, (c) type lc, and (d) type Id. Bottom: average velocity profiles in the vicinity of the inter-region obtained from direct numerical simulations (DNS) and with the one-domain approach (ODA) for the four variants of the type I geometry shown at the top. In all the simulations $\operatorname{Re}=10^{3}$ and $\xi \lambda^{*}=0.01$.

- Regarding the results for the first variant, the structure geometry plays a determining role in the maximum velocity values at the middle of the fracture. This contrasts with the values for the second and third variants. Note that some cylinders are cut for all the structures with the first variant. In specific, for the type I geometry, the average velocity is smaller than for the second and third variants because the upper and lower half cylinders constitute a significant flow resistance. To simplify, one may explain this velocity contrast by the fact that the aperture of the equivalent flat fracture is smaller for the first variant than for the second and third ones. The opposite is observed in the type II geometry and this is consistent with the fact that the flow paths are more complex for structures of types IIb and IIc, while it is mostly straight in structure of type-IIa. Again, this can be simply summarized by noticing that the aperture of the equivalent flat fracture is smaller in structures of types IIb and IIc than in IIa. Finally, for the random geometries, it turns out that the average velocity in the first variant is only slightly smaller than that for the second and third variants because only few cylinders were cut 


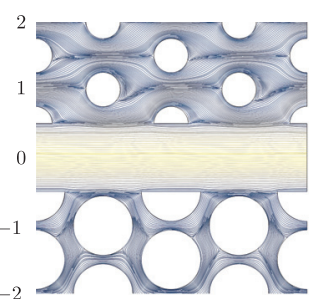

a)

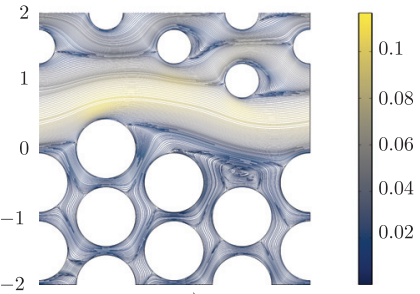

c)

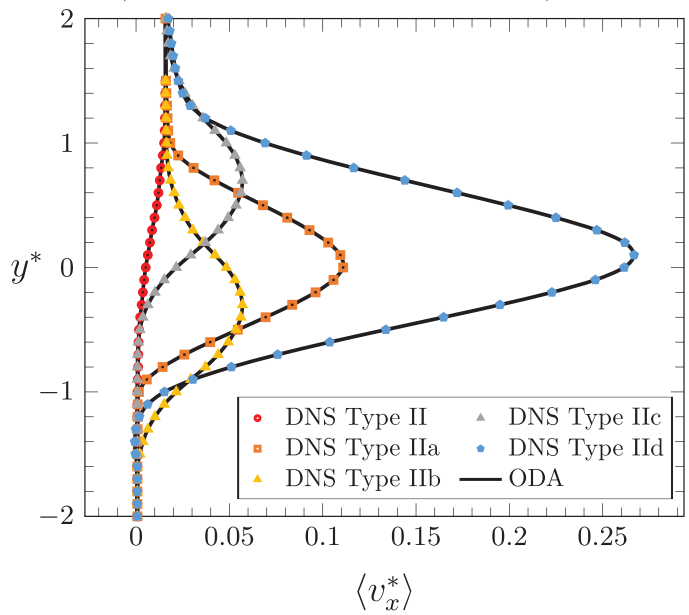

FIG. 6. Top: examples of the streamlines pattern colored by the dimensionless velocity magnitude near the frontier of two porous media separated by a fracture. The structures correspond to the four variants considered for the type II: (a) type $\mathrm{Ila}$, (b) type Ilb, (c) type Ilc, and (d) type Ild. Bottom: average velocity profiles in the vicinity of the inter-region obtained from direct numerical simulations (DNS) and with the one-domain approach (ODA) for the four variants of the type II geometry shown at the top. In all the simulations $\operatorname{Re}=10^{3}$ and $\xi \lambda^{*}=0.01$.

in the type-IIIa geometry. The same arguments in terms of the equivalent flat fracture aperture also explain this behavior.

As a final note, it is worth highlighting the fact that the agreement between the predictions from the ODA and the DNS is excellent. This serves to conclude that the model derived here is applicable when two porous media are separated by a fracture.

\section{Case 3: A porous medium in contact with a solid}

The following case study is the flow in the vicinity of a porous medium and a flat or rough wall. The wall roughness considered in this case and in the following one consists in a sinusoidal oscillation with a period corresponding to that of the geometrical unit cell. Three amplitudes were considered for the wall roughness, namely, $0.1 \ell_{c}$

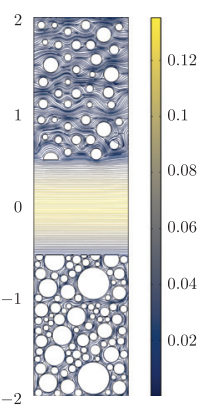

a)

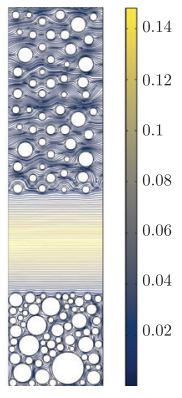

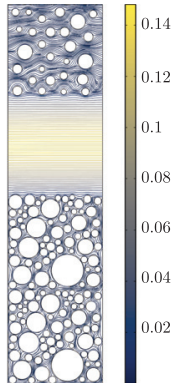
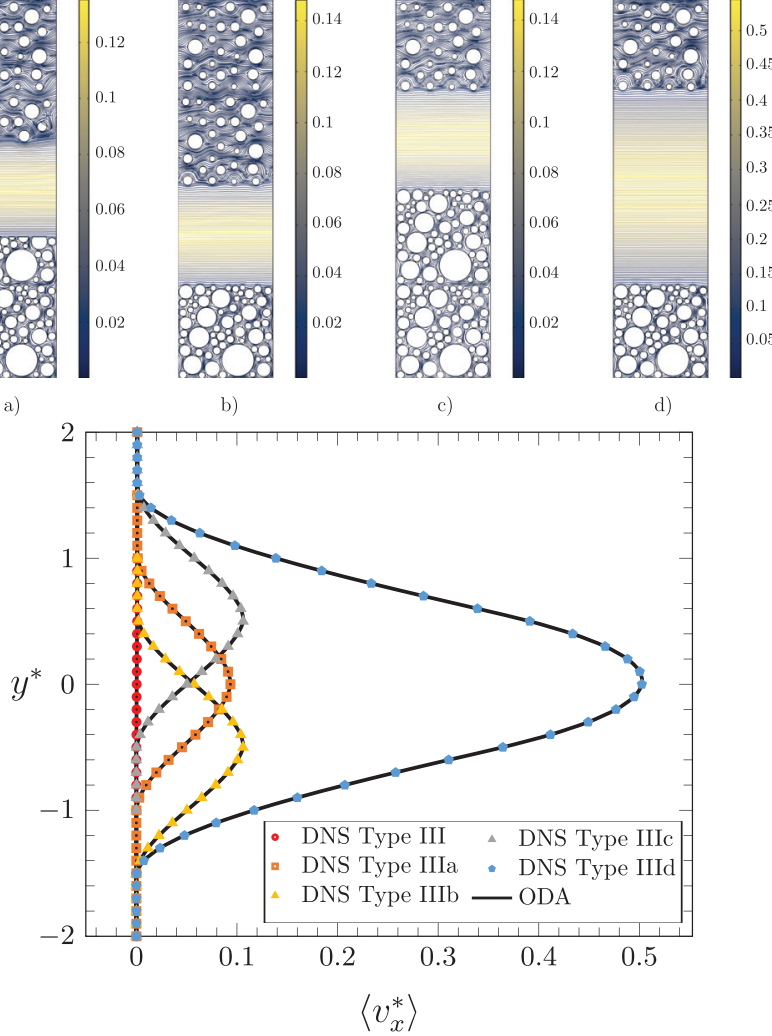

FIG. 7. Top: examples of the streamlines pattern colored by the dimensionless velocity magnitude near the frontier of two porous media separated by a fracture. The structures correspond to the four variants considered for the type III: (a) type IIla, (b) type IIIb, (c) type IIIc, and (d) type IIId. Bottom: average velocity profiles in the vicinity of the inter-region obtained from direct numerical simulations (DNS) and with the one-domain approach (ODA) for the four variants of the type III geometry shown at the top. In all the simulations $R e=10^{3}$ and $\xi \lambda^{*}=0.01$.

(sinusoidal-I), $0.2 \ell_{c}$ (sinusoidal-II), and $0.3 \ell_{c}$ (sinusoidal-III). For the remainder of this section, the porosity in the $\omega_{2}$-region is fixed to be $\varepsilon_{\omega_{2}}=0.8$ and only the structures of types I and II are analyzed. The results are reported in Figs. 8 and 9, respectively. As expected, when the averaging domain does not contain any fluid, the average velocity is zero. Subsequently, the velocity increases in a different fashion depending on the porous geometry and the type of wall roughness. As shown in Fig. 8, for the type I geometry, a step change in the velocity is observed that is centered around $y=0$, with the velocity increasing as the wall roughness is decreased. These noticeable vertical average velocity gradients are attenuated as $y$ increases until converging to the constant average velocity value in the homogeneous $\omega_{2}$-region when the centroid of the averaging domain is located near $y=1.5 \ell_{c}$.

Directing the attention to the results represented in Fig. 9, it is striking that the step-like change in the average velocity profiles is not present around $y=0$, but in the upper zone, near $y=0.6 \ell_{c}$. This is where the largest average velocity values are reached for the flat and sinusoidal-I structures. Nevertheless, the average velocity profiles follow the same trend as those corresponding to the type-I structure, i.e., the largest values correspond to the flat wall and they decrease as the 


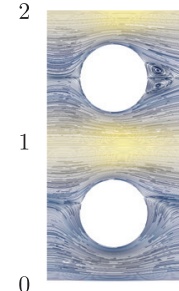

a)

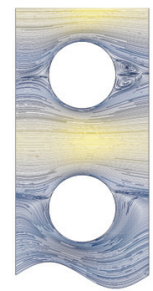

b)

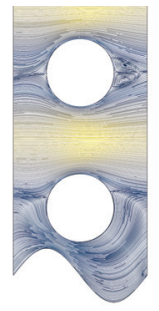

c)

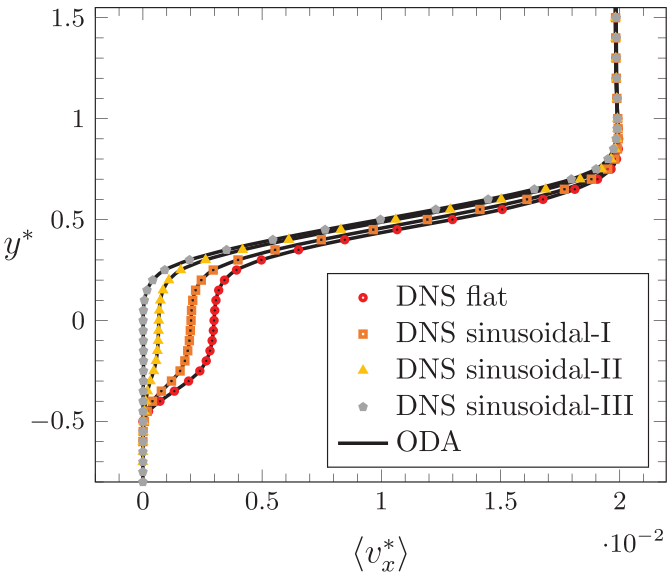

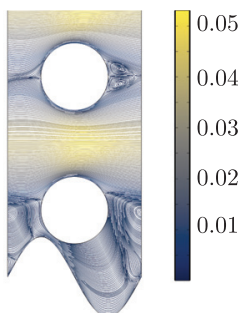

d)
0

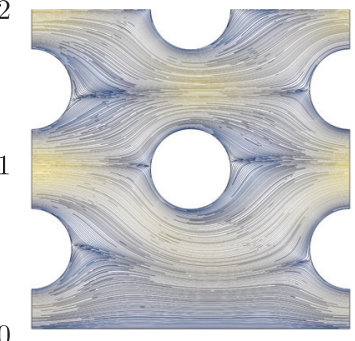

a)

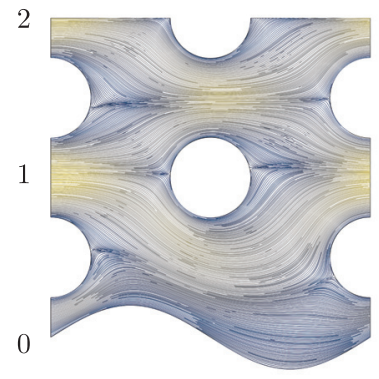

c)

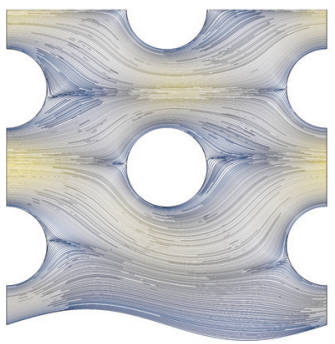

b)

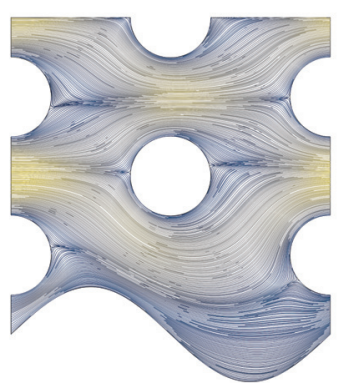

d)

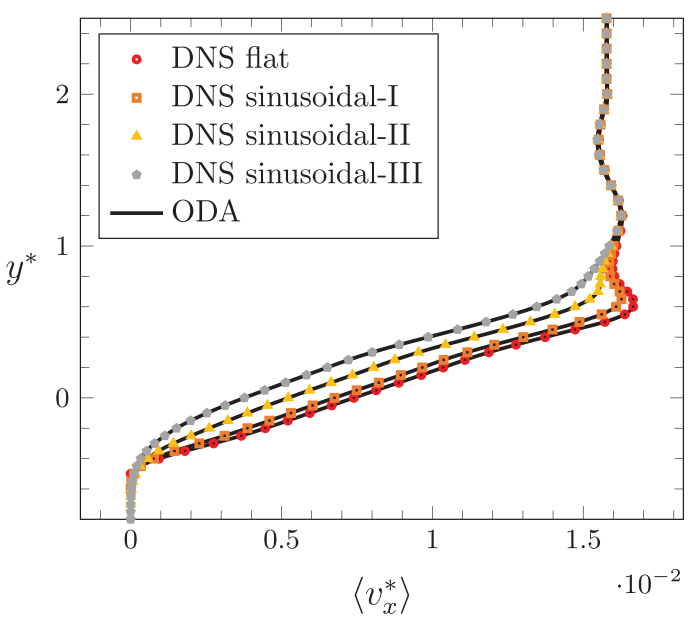

FIG. 9. Top: examples of the streamlines pattern colored by the dimensionless velocity magnitude near the frontier between a porous medium composed of staggered cylinders and a wall. The wall geometries correspond to (a) a flat wall, (b) a sinusoidal wall with amplitude of $0.1 \ell_{c}$ (sinusoidal-I), (c) a sinusoidal wall with amplitude of $0.2 \ell_{c}$ (sinusoidal-II), and (d) a sinusoidal wall with amplitude of $0.3 \ell_{c}$ (sinusoidal-III). Bottom: average velocity profiles resulting from direct numerical simulations (DNS) and the one-domain approach (ODA). In all cases $\varepsilon=0.8$, $R e=1000$, and $\xi \lambda^{*}=0.01$.

\section{Case 4: A porous medium and a solid separated by a fluid channel}

The final case to discuss in this section corresponds to a porous medium in the vicinity of an impervious wall separated by a fluid channel. For the sake of brevity in presentation, only the results corresponding to the type-II geometry are reported because those for the type-I geometry were observed to be quite similar to those shown in Fig. 10. In this figure, the average velocity profiles, obtained from DNS and from the upscaled model, are reported, considering both a flat and the three amplitudes of the sinusoidal wall presented in the previous case. This configuration is similar to the classical one studied by Beavers and Joseph, and this is confirmed by the shape of the average velocity profiles reported in Fig. $10 .{ }^{10}$ Clearly, the largest average velocity values are observed when the impervious wall is flat and noticeably decrease as the amplitude of the wall roughness increases. As in the previous case, this is partly due to the recirculation zones in the valleys of the wall that induce a significant energy loss. Note that the location of the maximum average velocity changes with the measure of the wall 


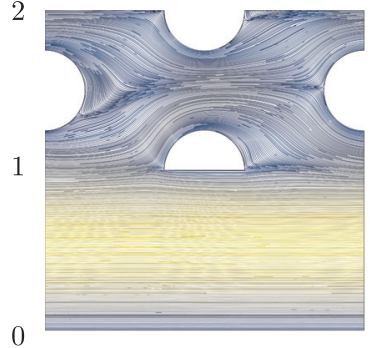

a)

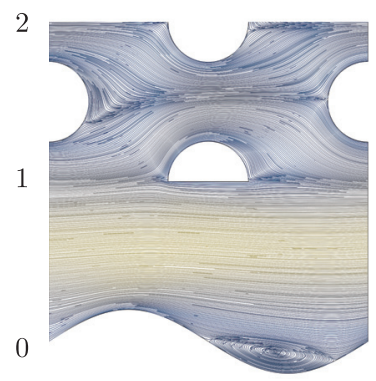

c)

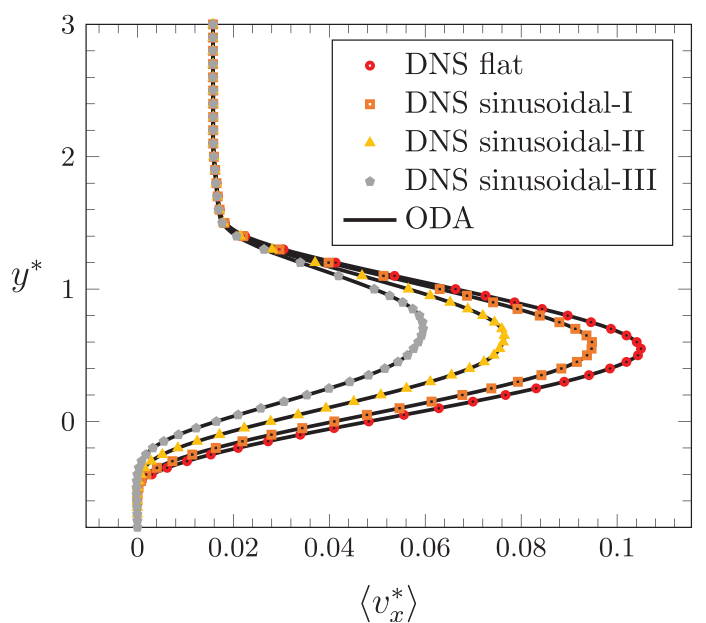

FIG. 10. Average velocity profiles between a porous medium and a wall separated by a fracture of height $\ell_{c}$ considering arrays of staggered cylinders. The results correspond to the solution of the pore-scale equations using direct numerical simulations (DNS) and the one-domain approach (ODA). The wall was considered flat and also sinusoidal with amplitudes of $0.1 \ell_{c}$ (sinusoidal-I), $0.2 \ell_{c}$ (sinusoidal-II), and $0.3 \ell_{c}$ (sinusoidal-III). In all cases $\varepsilon=0.8, R e=1000$, and $\xi \lambda^{*}=0.01$.

roughness. Finally, in all the cases, the values of the average velocity in the homogeneous $\omega_{2}$-region of the porous medium are recovered at $y \simeq 2 \ell_{c}$. Again, the agreement between the predictions from the ODA and the DNS data is excellent, thus validating the upscaled model in this final case study.

\section{E. Finite-size structure}

The perfect match between the DNS results and the macroscopic model for all the cases reported above was indeed expected due to the infinite periodic structures making the average model from the ODA exact in that case. However, agreement shall be further investigated when the system has a finite extension in the $x$-direction using Dirichlet pressure boundary conditions instead of periodicity. For this purpose, the DNS may now be conceived as numerical experiments in a domain made of an $M$-repetition of a vertical stripe of unit cells as the one sketched in Fig. 2. This analysis, carried out for one type of structure in each of the four cases investigated above, is reported in the Appendix. It shows that the agreement remains excellent far enough from the macroscopic boundaries, i.e., at a distance where the influence of the Dirichlet boundary conditions, that are not compatible with periodicity in the general case, becomes insensitive. The results indicate that the value of $M$ to reach a relative difference smaller than $1 \%$ between the macroscopic model prediction and DNS at $x^{*}=M / 2$ (taking the ODA as the reference) strongly depends on the configuration under consideration. It can be concluded that, under these circumstances, the ODA developed here perfectly captures the mean flow. Certainly, this conclusion is not restricted to the geometrical configurations reported here. As a matter of fact, on the basis of the results reported above, it is reasonable to propose that, as long as the assumptions involved in the model derivation are met, the ODA should provide reliable predictions of the average velocity in any other type of inter-region.

\section{CONCLUSION}

In this work, a general macroscopic model describing one-phase, steady, incompressible, inertial, and isothermal flow of a Newtonian fluid in the vicinity of porous medium boundaries was derived. The different configurations studied here included flow between two porous media, or a porous medium and a solid, the two being nondeformable and in direct contact or separated by a fluid-saturated fracture, the boundaries being flat or wavy. The contribution of slip effects at the solid-fluid interfaces was taken into account in the physical process.

A one-domain approach was followed and a simplified version of the volume averaging method was employed to carry out the upscaling on the pore-scale boundary value problem (Navier-Stokes and firstorder slip boundary condition). Conceiving the system as being composed of two homogeneous regions and an inter-region, the procedure yielded a unified macroscopic model under constraints that were made clear in the course of the development. In particular, the classical separation of length-scales and constant macroscopic pressure gradients are pre-requisites for the derivations presented in this work to be valid. The upscaled model consists of a divergence-free filtration velocity for the mass balance equation and a unique momentum equation having the structure of Darcy's law, valid in the entire system. In this macroscopic momentum balance, the apparent permeability tensors are constant valued in the homogeneous regions but space-dependent in the inter-region, accounting for the changes of the structure (and hence of the flow) in the vicinity of the boundaries. The permeability tensors can be computed from the solution of coupled closure problems that have a physical structure similar to the original pore-scale problem. This simple mathematical structure of the upscaled model makes it appealing for its application in a wide variety of practical situations.

To illustrate the upscaled model performance, a set of twodimensional case studies was considered, including (i) two porous media in direct contact with a flat or wavy boundary, or with a continuously evolving porosity from one homogeneous region to the other; 
(ii) two porous media separated by a flat or wavy fluid channel (a fracture); (iii) a porous medium in direct contact with a flat or wavy solid impervious wall; and (iv) a porous medium separated from a solid impervious wall by a flat or wavy fluid channel. In all cases, an excellent agreement was obtained between the average velocity profiles predicted by the one-domain average model and those computed from direct numerical simulations of the pore-scale problem in the whole system.

The one-domain approach developed in this work provides an efficient, simple, and versatile macroscopic model that is an interesting alternative to existing one- or two-domain approaches reported in the literature. The proposed methodology is promising and may be applied for the study of more complicated situations, such as multiphase and even non-isothermal flow near porous media boundaries. These, and other applications, will be studied in future works.

\section{ACKNOWLEDGMENTS}

D.L. acknowledges the support from ANR through Project No. ANR-11-RSNR-0012-01, within which part of this work was accomplished.

\section{APPENDIX: DNS IN FINITE-SIZE SYSTEMS}

In this appendix, results obtained from DNS conceived as numerical experiments over domains of finite size are compared to the predictions of the macroscopic model. In contrast to the DNS reported in Sec. IV where media were considered as periodic and infinite in the $x$-direction, the system is supposed to be made of an $M$-repetition of a vertical stripe of unit cells as the one sketched in Fig. 2. Instead of periodic boundary conditions, pressure Dirichlet conditions are considered at the entrance $\left(x^{*}=0\right)$ and exit $\left(x^{*}=M\right)$. Moreover, walls with a slip velocity condition are assumed at $y^{*}=y_{\omega_{i}}^{*}=10, i=1,2$. The latter was chosen in agreement with the observation from the DNS results presented in Sec. IV showing that, at this distance, the boundary condition has no impact on the flow in the $\omega$-region.

With the dimensionless form adopted in this work, the flow problem to be solved for these numerical experiments is given by

$$
\nabla^{*} \cdot \mathbf{v}_{\kappa}^{*}=0 \text { in the } \beta \text {-phase, }
$$

$$
\operatorname{Re} \mathbf{v}_{\kappa}^{*} \cdot \nabla^{*} \mathbf{v}_{\kappa}^{*}=-\nabla^{*} p_{\kappa}^{*}+\nabla^{* 2} \mathbf{v}_{\kappa}^{*} \quad \text { in the } \beta \text {-phase, }
$$

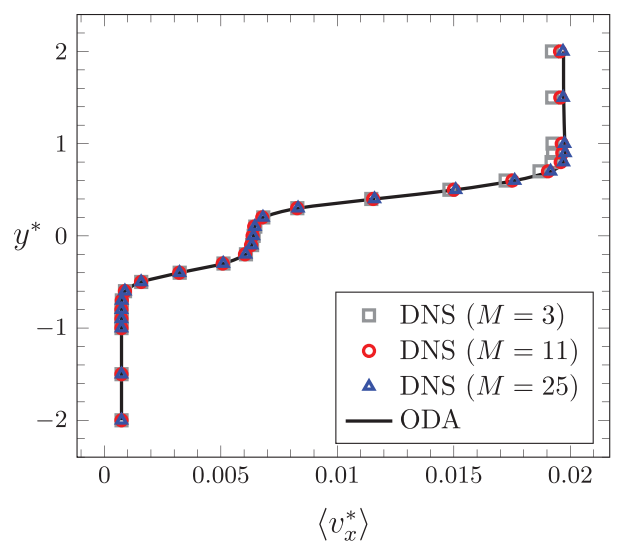

a)

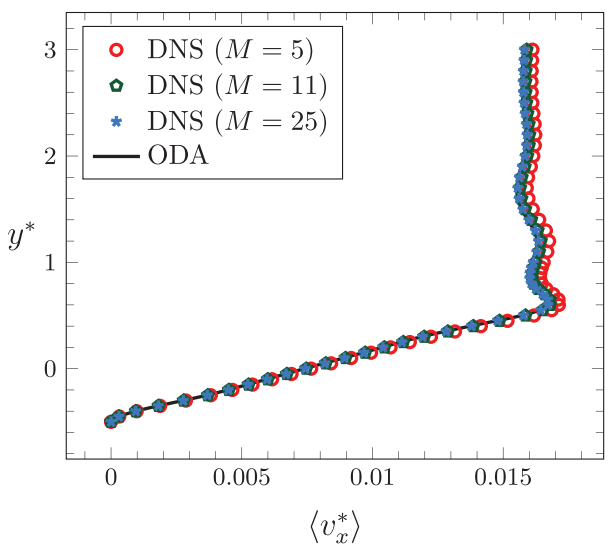

c)

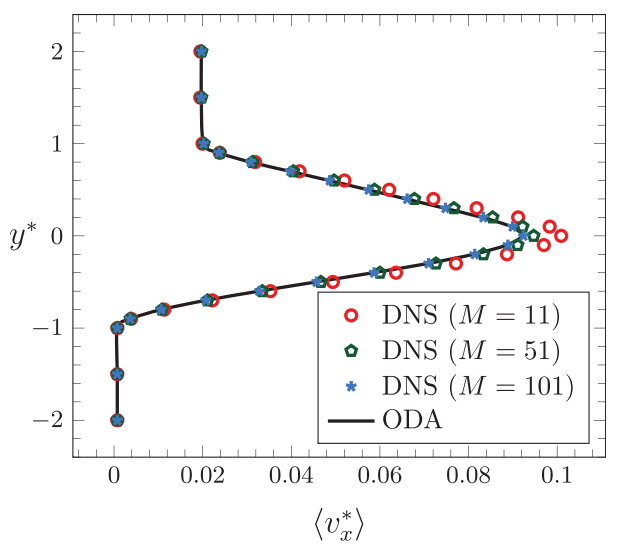

b)

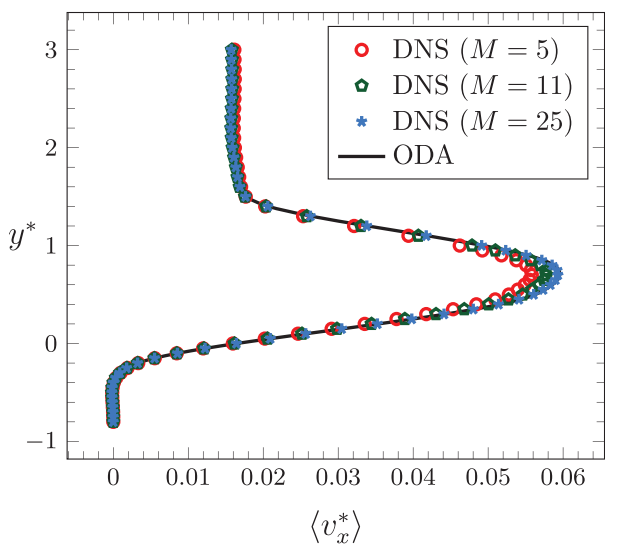

d)
FIG. 11. Examples of the convergence of the $y^{*}$ profiles of $\left\langle v^{*}\right\rangle$ resulting from direct numerical simulations (DNS) in systems of different sizes in the $x$-direction toward those predicted from the average model derived with the ODA. The size of the system is $M \ell_{c}$. Results correspond to (a) case 1, type I, (b) case 2, type la, (c) case 3 , flat and (d) case 4 , sinusoidal-III in the main text. 


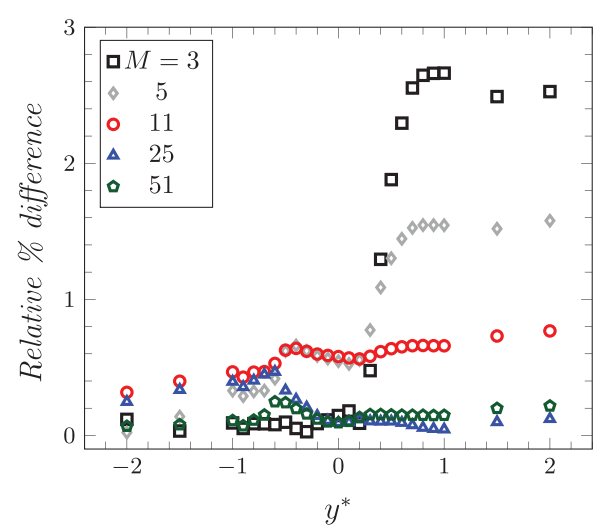

a)

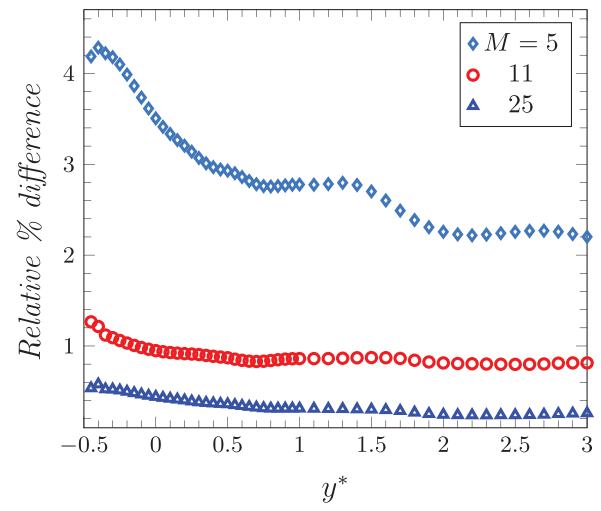

c)

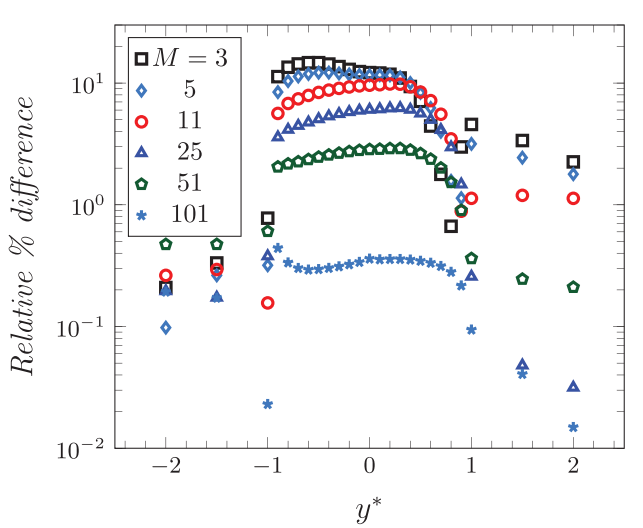

b)

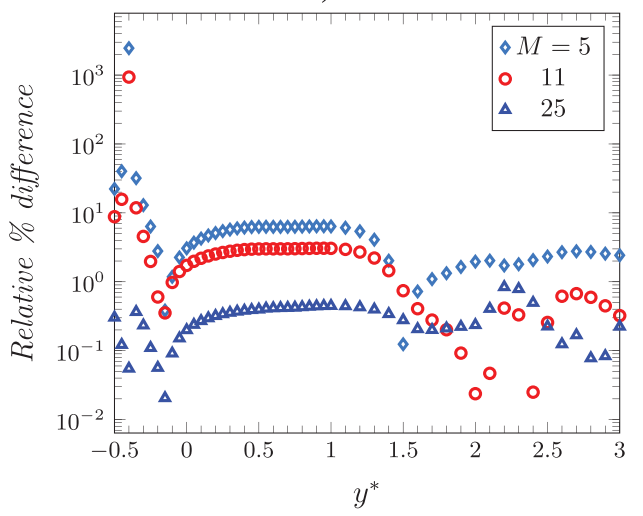

d)
FIG. 12. Relative percent difference of the DNS results on $\left\langle v_{x}^{*}\right\rangle$ with respect to the ODA for the results reported in Fig. 11.

$$
\begin{gathered}
\mathbf{v}_{\kappa}^{*}=-\xi_{i} \lambda^{*}(\mathbf{I}-\mathbf{n n}) \cdot\left[\mathbf{n} \cdot\left(\nabla^{*} \mathbf{v}_{\kappa}^{*}+\nabla^{*} \mathbf{v}_{\kappa}^{* T}\right)\right] \text { at } \mathscr{A}_{\beta \sigma_{i}}^{\kappa}, \quad i=1,2, \\
p^{*}=M, \quad \text { at } x^{*}=0 ; \quad p^{*}=0 \quad \text { at } x^{*}=M, \\
\mathbf{v}_{\kappa}^{*}=-\xi_{i} \lambda^{*}(\mathbf{I}-\mathbf{n n}) \cdot\left[\mathbf{n} \cdot\left(\nabla^{*} \mathbf{v}_{\kappa}^{*}+\nabla^{*} \mathbf{v}_{\kappa}^{* T}\right)\right] \text { at } y_{\omega_{i}}^{*} .
\end{gathered}
$$

Since the objective is not to report about this analysis in all the different configurations considered in Sec. IV, only a few illustrative ones were selected, namely, case 1, type I; case 2, type Ia; case 3, flat and case 4, sinusoidal-III. The DNS were performed with several values of $M$ to analyze the convergence of the results toward the predictions from the ODA and the relative \% difference between the two was computed at $x^{*}=M / 2$, taking the ODA as the reference. In Fig. 11, the profiles of $\left\langle v_{x}^{*}\right\rangle$ are represented vs $y^{*}$ at $x^{*}=M / 2$ for the different values of $M$. In the four situations, the prediction from the ODA either overestimates (cases 1 and 4) or underestimates (cases 2 and 3 ) the average velocity obtained from DNS. In addition, the length of the domain for which the prediction of the average velocity from the ODA can be achieved with a given accuracy depends on the configuration under consideration. Indeed, as shown in Fig. 12 representing the relative \% difference between the two approaches, if a criterion of $1 \%$ accuracy is chosen, then $M=11$ is sufficient in case 1 , whereas $M=25$ is necessary in cases 3 and 4 , and, for case $2, M=101$ is required. The reason for this is due to the fact that case 1 corresponds to a situation with the largest viscous drag, followed by cases 3,4 , and 2; hence, the distance after which periodic boundary conditions can be used increases with the reduction of the drag, as expected. The conclusion from this analysis is that convergence can be reached provided $M$ is large enough, the value of $M$ at convergence strongly depending on the structure and the type of inter-region under consideration. This was confirmed by simulations performed in many other cases investigated in Sec. IV that are however not reported here for the sake of brevity.

\section{DATA AVAILABILITY}

The data that support the findings of this study are available from the corresponding author upon reasonable request.

\section{REFERENCES}

'G. Beavers and D. Joseph, "Boundary conditions at a naturally permeable wall," J. Fluid Mech. 30, 197 (1967).

${ }^{2}$ A. Mikelić and W. Jäger, "On the interface boundary condition of Beavers, Joseph, and Saffman," SIAM J. Appl. Math. 60, 1111-1127 (2000).

${ }^{3} \mathrm{M}$. Chandesris and D. Jamet, "Boundary conditions at a planar fluid-porous interface for a Poiseuille flow," Int. J. Heat Mass Transfer 49, 2137-2150 (2006).

${ }^{4} \mathrm{~W}$. Jäger and A. Mikelić, "Modeling effective interface laws for transport phenomena between an unconfined fluid and a porous medium using homogenization," Transp. Porous Media 78, 489-508 (2009). 


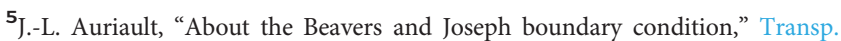
Porous Media 83, 257-266 (2010).

${ }^{6} \mathrm{D}$. A. Nield, "The Beavers-Joseph boundary condition and related matters: A historical and critical note," Transp. Porous Media 78, 537-540 (2009).

${ }^{7} \mathrm{~W}$. Jäger and A. Mikelić, "Letter to the editor: Comments on 'About the Beavers and Joseph boundary condition,' DOI:10.1007/s11242-009-9435-9," Transp. Porous Media 83, 267-268 (2010).

${ }^{8} \mathrm{~J}$.-L. Auriault, "Reply to the comments on 'About the Beavers and Joseph boundary condition," Transp. Porous Media 83, 269-270 (2010).

${ }^{9}$ A. Samanta, "Role of slip on the linear stability of a liquid flow through a porous channel," Phys. Fluids 29, 094103 (2017).

${ }^{10} \mathrm{~F}$. J. Valdés-Parada and D. Lasseux, "A novel one-domain approach for modeling flow in a fluid-porous system including inertia and slip effects," Phys. Fluids 33, 022106 (2021).

${ }^{11}$ A. Goharzadeh, A. Khalili, and B. B. Jørgensen, "Transition layer thickness at a fluid-porous interface," Phys. Fluids 17, 057102 (2005).

${ }^{12} \mathrm{M}$. R. Morad and A. Khalili, "Transition layer thickness in a fluid-porous medium of multi-sized spherical beads," Exp. Fluids 46, 323-330 (2009).

${ }^{13}$ A. Terzis, I. Zarikos, K. Weishaupt, G. Yang, X. Chu, R. Helmig, and B. Weigand, "Microscopic velocity field measurements inside a regular porous medium adjacent to a low Reynolds number channel flow," Phys. Fluids 31, 042001 (2019).

${ }^{14}$ C. Guo, Y. Li, X. Nian, M. Xu, H. Liu, and Y. Wang, "Experimental study on the slip velocity of turbulent flow over and within porous media," Phys. Fluids 32, 015111 (2020).

${ }^{15} \mathrm{M}$. Sahraoui and M. Kaviany, "Slip and no-slip velocity boundary conditions at interface of porous, plain media," Int. J. Heat Mass Transfer 35, 927-943 (1992).

${ }^{16} \mathrm{~A}$. Nabovati and C. Amon, "Hydrodynamic boundary condition at openporous interface: A pore-level lattice Boltzmann study," Transp. Porous Media 96, 83-95 (2013).

${ }^{17} \mathrm{H}$. Chen and X.-P. Wang, "A one-domain approach for modeling and simulation of free fluid over a porous medium," J. Comput. Phys. 259, 650-671 (2014).

${ }^{18} \mathrm{E}$. Eggenweiler and I. Rybak, "Unsuitability of the Beavers-Joseph interface condition for filtration problems," J. Fluid Mech. 892, A10 (2020).

${ }^{19}$ R. E. C. P. Meira, F. C. D. Lai, C. O. R. Negrão, and S. L. M. Junqueira, "On determining the power-law fluid friction factor in a partially porous channel using the lattice Boltzmann method," Phys. Fluids 32, 093104 (2020).

${ }^{20}$ I. Rybak, C. Schwarzmeier, E. Eggenweiler, and U. Rüde, "Validation and calibration of coupled porous-medium and free-flow problems using pore-scale resolved models," Comput. Geosci. 25, 621-635 (2021).

${ }^{21} \mathrm{~W}$. Jäger and A. Mikelić, "On the flow conditions at the boundary between a porous medium and an impervious solid," in Progress in Partial Differential Equations: The Metz Surveys 3 (CRC Press, 1994), pp. 145-160.

${ }^{22}$ S. Haber and R. Mauri, "Boundary conditions for Darcy's flow through porous media," Int. J. Multiphase Flow 9, 561-574 (1983).

${ }^{23}$ J. Ochoa-Tapia, F. Valdés-Parada, B. Goyeau, and D. Lasseux, "Fluid motion in the fluid/porous medium inter-region," Rev. Mex. Ing. Quím. 16, 923-938 (2017), http://rmiq.org/ojs311/index.php/rmiq/article/view/98.

${ }^{24} \mathrm{M}$. Quintard and S. Whitaker, "Écoulement monophasique en milieu poreux: Effet des hétérogénéités locales," J. Méc. Théor. Appl. 6, 691-726 (1987), https://www. researchgate.net/publication/230704130_Ecoulement_monophasique en milieu. poreux_Effet_des_heterogeneites_locales.
${ }^{25}$ P. M. Adler, J.-F. Thovert, and, and V. V. Mourzenko, Fractured Porous Media (Oxford University Press, 2012).

${ }^{26}$ I. Berre, F. Doster, and E. Keilegavlen, "Flow in fractured porous media: A review of conceptual models and discretization approaches," Transp. Porous Media 130, 215-236 (2019).

${ }^{27} \mathrm{G}$. Zampogna and F. Gallaire, "Effective stress jump across membranes," J. Fluid Mech. 892, A9 (2020)

${ }^{28}$ J. Feng and S. Weinbaum, "Lubrication theory in highly compressible porous media: The mechanics of skiing, from red cells to humans," J. Fluid Mech. 422, 281-417 (2000).

${ }^{29} \mathrm{Z}$. Zhu, R. Nathan, and Q. Wu, "Multi-scale soft porous lubrication," Tribol. Int. 137, 246-253 (2019).

${ }^{30} \mathrm{Z}$. Kou and M. Dejam, "Dispersion due to combined pressure-driven and electro-osmotic flows in a channel surrounded by a permeable porous medium," Phys. Fluids 31, 056603 (2019).

${ }^{31}$ D. Einzel, P. Panzer, and M. Liu, "Boundary condition for fluid flow: Curved or rough surfaces," Phys. Rev. Lett. 64, 2269-2272 (1990).

${ }^{32}$ R. Barber, Y. Sun, X. Gu, and D. Emerson, "Isothermal slip flow over curved surfaces," Vacuum 76, 73-81 (2004).

${ }^{33} \mathrm{E}$. Lauga, M. Brenner, and H. Stone, "Microfluidics: The no-slip boundary condition," in Springer Handbook of Experimental Fluid Mechanics (Springer, Berlin/Heidelberg, 2007), pp. 1219-1240.

${ }^{34}$ S. Pasquier, M. Quintard, and Y. Davit, "Modeling flow in porous media with rough surfaces: Effective slip boundary conditions and application to structured packings," Chem. Eng. Sci. 165, 131-146 (2017).

${ }^{35} \mathrm{E}$. Sanchez-Palencia, Non-Homogeneous Media and Vibration Theory, Lecture Notes in Physics Vol. 127 (Springer, Berlin/Heidelberg, 1980).

${ }^{36}$ S. Whitaker, The Method of Volume Averaging (Springer, 1999).

${ }^{37} \mathrm{~F}$. A. Howes and S. Whitaker, "The spatial averaging theorem revisited," Chem. Eng. Sci. 40, 1387-1392 (1985).

${ }^{38}$ W. Gray and C. Miller, "A generalization of averaging theorems for porous medium analysis," Adv. Water Resour. 62, 227-237 (2013).

${ }^{39} \mathrm{~S}$. Whitaker, "The Forchheimer equation: A theoretical development," Transp. Porous Media 25, 27-61 (1996).

${ }^{40}$ D. Lasseux, F. J. Valdés-Parada, J. A. Ochoa-Tapia, and B. Goyeau, "A macroscopic model for slightly compressible gas slip-flow in homogeneous porous media," Phys. Fluids 26, 053102 (2014).

${ }^{41}$ D. Lasseux, F. J. Valdés-Parada, and M. L. Porter, "An improved macroscale model for gas slip flow in porous media," J. Fluid Mech. 805, 118-146 (2016).

${ }^{42}$ D. Lasseux, F. J. Valdés-Parada, and F. Bellet, "Macroscopic model for unsteady flow in porous media," J. Fluid Mech. 862, 283-311 (2019).

${ }^{43}$ J. Bear, Modeling Phenomena of Flow and Transport in Porous Media (Springer International Publishing, 2018).

${ }^{44}$ W. G. Gray, "A derivation of the equations for multi-phase transport," Chem. Eng. Sci. 30, 229-233 (1975).

${ }^{45} \mathrm{~A}$. Bottaro, "Flow over natural or engineered surfaces: An adjoint homogenization perspective," J. Fluid Mech. 877, P1 (2019).

${ }^{46} \mathrm{D}$. Lasseux and F. J. Valdés-Parada, "Symmetry properties of macroscopic transport coefficients in porous media," Phys. Fluids 29, 043303 (2017).

${ }^{47} \mathrm{M}$. Agnaou, D. Lasseux, and A. Ahmadi, "From steady to unsteady laminar flow in model porous structures: An investigation of the first Hopf bifurcation," Comput. Fluids 136, 67-82 (2016). 